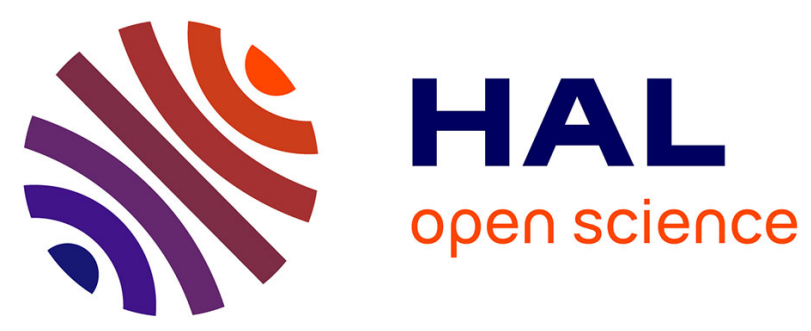

\title{
Hybrid Navigation Filters Performances Between GPS, Galileo and 5G TOA Measurements in Multipath Environment
}

\author{
Anne-Marie Tobie, Axel Javier Garcia Peña, Paul Thevenon, Jérémy Vezinet, \\ Marion Aubault-Roudier
}

\section{To cite this version:}

Anne-Marie Tobie, Axel Javier Garcia Peña, Paul Thevenon, Jérémy Vezinet, Marion AubaultRoudier. Hybrid Navigation Filters Performances Between GPS, Galileo and 5G TOA Measurements in Multipath Environment. ION GNSS+ 2020, 33rd International Technical Meeting of the Satellite Division of the Institute of Navigation, Sep 2020, Virtual event, United States. pp. 2107-2140., 10.33012/2020.17521 . hal-02963979

\section{HAL Id: hal-02963979 \\ https://hal-enac.archives-ouvertes.fr/hal-02963979}

Submitted on 30 Nov 2020

HAL is a multi-disciplinary open access archive for the deposit and dissemination of scientific research documents, whether they are published or not. The documents may come from teaching and research institutions in France or abroad, or from public or private research centers.
L'archive ouverte pluridisciplinaire HAL, est destinée au dépôt et à la diffusion de documents scientifiques de niveau recherche, publiés ou non, émanant des établissements d'enseignement et de recherche français ou étrangers, des laboratoires publics ou privés. 


\title{
Hybrid Navigation Filters Performances Between GPS, Galileo and 5G TOA Measurements in Multipath Environment
}

\author{
Anne-Marie TOBIE, TéSA \\ Axel GARCIA-PENA, Paul THEVENON, Jérémy VEZINET, ENAC Université de Toulouse \\ Marion AUBAULT, CNES the French Space Agency
}

\section{BIOGRAPHIES}

Anne-Marie TOBIE received the M.Sc. degree in aero-nautical and space telecommunications engineering from ENAC (French Civil Aviation University), Toulouse, France, in 2017. Since 2017, she is a PhD student in the Signal Processing and Navigation (SIGNAV) research axis of the TELECOM team at the ENAC. She is working on 5G and GNSS hybridization for urban positioning.

Axel GARCIA-PENA is a researcher/lecturer with the SIGnal processing and NAVigation (SIGNAV) research axis of the TELECOM lab of ENAC (French Civil Aviation University), Toulouse, France, since February 2011. His research interests are GNSS signal and receiver design, GNSS satellite payload analysis and navigation with SoO . He received his double engineer degree in 2006 in digital communications from SUPAERO and UPC, and his PhD in 2010 from the Department of Mathematics, Computer Science and Telecommunications of the INPT (Polytechnic National Institute of Toulouse), France.

Paul THEVENON graduated as electronic engineer from Ecole Centrale de Lille in 2004 and obtained in 2007 a research master at ISAE in space telecommunications. In 2010, he obtained a PhD degree in the signal processing laboratory of ENAC in Toulouse, France. From 2010 to 2013, he was employed by CNES, the French space agency, to supervise GNSS research activities and measurement campaigns. Since July 2013, he is employed by ENAC as Assistant Professor. His current activities are GNSS signal processing, GNSS integrity monitoring and hybridization of GNSS with other sensors.

Marion AUBAULT-ROUDIER is a Radionavigation engineer in the navigation/location signals department at CNES, the French Space Agency, where she is involved in the optimization of GNSS signals. She graduated as an electronics engineer in 2011 from ENAC (Ecole Nationale de l'Aviation Civile) in Toulouse, France. She received her PhD in 2015 from the Department of Mathematics, Computer Science and Telecommunications of the INPT (Polytechnic National Institute of Toulouse), France.

Jérémy VEZINET holds a Ph.D. in multi-sensors hybridization. He is a Research Associate in the TELECOM team of ENAC in Toulouse (France) since 2014. He has been involved in several projects on multi-antenna GNSS receivers, GNSS/INS integration techniques and integrity monitoring. His research interests are GNSS, Inertial Navigation, Multi-sensor Hybridization, Integrity Monitoring and Video-Based Navigation.

\section{ABSTRACT}

In this paper, the performance of different hybrid navigation filters exploiting GPS, Galileo and 5G Time Of Arrival (TOA) measurements in multipath environment are studied. For the realism of the study, realistic propagation channels must be considered and their impacts on the received signals processing must be accurately modelled. GNSS signal mathematical models in multipath environment have been analyzed for a long time. However, 5G mathematical models in a realistic multipath environment are still in its early stages of analysis. This article is divided in three main parts.

The first part is dedicated to the identification of compliant GNSS and 5G signal propagation channel models; SCHUN is selected for GNSS and QuaDRiGa is selected for 5G. Based on this, the correlator output mathematical models for 5G signals and GNSS signals are derived.

The second part tackles the accurate characterization of the pseudo range errors due to propagation channels shadowing and multipath effect as well as thermal noise. This step is required for the correct derivation of the navigation filters. Indeed, the 
study will focus on Extended Kalman Filters (EKF) and Unscented Kalman Filters (UKF); both assume a Gaussian distribution of the errors. Therefore, by optimally characterizing the errors, the performances of the filters are expected to be improved.

The last part consists in validating through simulations the theory and mathematical models developed in the first and second parts.

\section{INTRODUCTION}

Over the past few years, the need for positioning, and thus the number of positioning services in general, has been in constant growth. Moreover, this need for positioning has been increasingly focused on constrained environments, such as urban or indoor environments, where GNSS (Global Navigation Satellite System) is known to have significant limitations: multipath as well as the lack of Line-of-Sight satellite visibility degrades the GNSS positioning solution and makes it unsuitable for some urban or indoor applications. In order to improve the positioning performance and availability in constrained environments, many solutions are already available: hybridization with additional sensors, [1], [2] or the use of signals of opportunity (SoO) for example, [3], [4], [5], [6]. [7], [8]. Concerning SoO, mobile communication signals, such as the 4G Long Term Evolution (LTE) or 5G, are naturally envisioned for positioning, [3], [9], [10]. Indeed, a significant number of users are expected to be "connected-users" and 5G systems offers promising opportunities.

In order to improve the positioning availability and GNSS positioning performance in urban environment through the exploitation of $5 \mathrm{G}$ signals, both systems, GNSS and 5G communication systems, must be optimally combined. In fact, in order to achieve this optimal combination, both types of signals must be optimally processed, and the mathematical model of their generated pseudo range measurements must be accurately characterized. However, even though GNSS systems are mature and a generic signal processing module is already known, $5 \mathrm{G}$ systems is a new technology, which is being standardized at 3rd Generation Partnership Project (3GPP, [11]). Therefore, its positioning performance, as well as a potential generic receiver scheme to conduct positioning operations, is still under analysis; this means that pseudo range measurements mathematical models, especially in urban canyons, remain to be derived. Moreover, note that in order to develop such a positioning module, the mathematical models of the processed 5G signals at each stage of the receiver must be perfectly characterized. These models have been derived in [12].

This article aims thus at realistically characterizing GNSS and 5G pseudo range measurement mathematical models and statistics and at developing hybrid navigation modules exploiting/adapted to the derived pseudo range measurements mathematical models. The pseudo range measurements mathematical models are derived from a realistic simulator which integrates a typical GNSS receiver processing module and a typical 5G signal processing module proposition; moreover, in order to achieve a realistic characterization, the simulator implements highly realistic propagation channels for GNSS, selected model is SCHUN [8], and for 5G, selected model is QuaDRiGa [9]. The hybrid navigation modules to be implemented and compared in this work are an Extended Kalman Filter (EKF) and an Unscented Kalman Filter (UKF).

The paper is organized in 7 sections. First the propagation channels used for both GNSS and 5G signals are presented. Second, highly realistic correlator output mathematical models are derived. Third, the pseudo range error distributions are characterized. Forth the implemented hybrid GNSS/5G navigation filters are presented. The simulator designed to test the previously derived mathematical models is presented in the fifth section. Finally, the results are presented.

\section{PROPAGATION CHANNEL PRESENTATION}

In this work the realism of the simulated signals is of the utmost importance; thus the selected 5G propagation channels must fulfil the 5G standard requirements defined in the 3GPP standard. GNSS propagation channel requirements are well known and subject to many studies in the literature. This section is divided in 2 subsections, first the presentation of the selected 5G propagation channel; second, the presentation of the adopted GNSS propagation channel.

The propagation channel impact on the transmitted signal is reminded in $(1)$, where $\alpha(t, \tau)$ is the time delay impulse response of the channel.

\section{1 - QuaDRiGa: a 5G compliant propagation channel}

$$
s_{\text {received signal }}(t)=s_{\text {transmitted signal }}(t) * \alpha(t, \tau)
$$

Two characteristics of the $5 \mathrm{G}$ signals must be kept in mind for the selection of a $5 \mathrm{G}$ compliant propagation channel. First, $5 \mathrm{G}$ systems aim at using several new technologies. Second, the range of use cases envisioned for $5 \mathrm{G}$ is much wider than with previous communication systems. Those characteristics make unsuitable propagation channels usually used for previous communication systems. First the innovations with $5 \mathrm{G}$ systems are briefly presented; second the adopted propagation channel is presented.

\subsubsection{G innovations}

In this sub section the $5 \mathrm{G}$ innovations are briefly presented; the objective here is to explain which characteristics of the system impact the modelling of the propagation channel. It also gives the readers the reference to study deeper these problematics.

When considering the use cases or scenarios envisioned for 5G, one should keep in mind that the main objective of 5G systems is to provide services to anyone and anything anywhere and anytime. In that perspective, $5 \mathrm{G}$ has to be available all the 
time and so a wide range of propagation scenarios have to be modelled. The wireless network has to serve from stationary users to mobile users, and the mobile-to-mobile links. The wireless system should work reliably in any environment, including indoor, outdoor-to-indoor, dense urban, wide area, highway, shopping mall, stadium, etc. The network topologies should support not only cellular, but also direct device-to-device (D2D), machine-to-machine (M2M) and vehicle-to-vehicle (V2V) links as well as full mesh networks. Thus, a huge range of case studies, not considered with previous communication systems, have to be considered.

Regarding the technologies, $5 \mathrm{G}$ systems aim at using several new technologies such as millimeter waves (mmW), massive Multiple Input Multiple Output (MIMO) antenna or denser network. From the spectral point of view, 5G aims at using mmW which are electromagnetic waves typically defined to lie within the frequency range of 30-300 GHz. Those frequencies are very promising; they make it possible to implement very small antennas and even massive array antennas in relatively small volume. In addition to that, the spectrum at mmW frequencies is rather unused, which means that wider bandwidths are available. However, the propagation properties for these frequencies are not perfectly known, [13].

Another important technological component of $5 \mathrm{G}$ mobile communications is the use of very large array antennas: massive MIMO. For these highly directive antennas or large array antennas huge performance are expected. However, these large arrays require non-planar wave modelling replacing the commonly used plane wave approximation. The use of Massive MIMO antenna is possible thanks to the use of millimeter waves, [14], [15].

Another technical direction of $5 \mathrm{G}$ systems is the use of denser network. Traditional networks use macro cells and microcells, with 5G systems pico-cells and femto-cells are expected to be use. Moreover, with 5G systems, scenarios where both ends of the links are moving (for example D2D scenario) are envisioned. The traffic is also expected to grow exponentially leading to a growing number of links. Hence, these features bring new requirements to channel modelling in terms of spatial consisten cy and mobility. The term spatial consistency means that the channel evolves smoothly without discontinuities when the transmitter and/or receiver moves or turns. It also means that channel characteristics are similar in closely located links.

In this subsection an insight of the new innovations envisioned for $5 \mathrm{G}$ has been provided. The presentation is not exhaustive but it proves the necessity to select an appropriate propagation channel adapted to the wide variety of case study and new technologies envisioned for $5 \mathrm{G}$.

\subsubsection{G compliant propagation channel}

A complete review of the literature on propagation channels has been made in order to select a proper propagation channel. The selected propagation channel had to fulfill several criteria; it had to be realistic, fast computing and it had to follow the 3GPP standards. In this article, QuaDRiGa, [9], a channel model developed at Fraunhofer HHI has been selected. This model proposes scenarios in accordance with the 3GPP standard on 5G signals and on 5G propagation channel. Here after a brief presentation of the model is provided.

The Quasi-Deterministic Radio channel Generator (QuaDRiGa) is a model evolved from the WINNER II project; it can be seen as a 3GPP 3D and 3GPP 38.901 reference implementation. It is used for generating realistic radio channel impulse responses for system-level simulations of mobile radio networks. QuaDRiGa was developed at Fraunhofer HHI to enable the modeling of MIMO radio channels for specific network configurations, such as indoor, satellite or heterogeneous configuration s. QuaDRiGa is a 3D-GSCM.

The QuaDRiGa channel model follows a geometry-based stochastic channel modeling (GSCM) approach, which allows the creation of an arbitrary double directional radio channel. The channel model is antenna independent, hence different antenna configurations and different element patterns can be inserted. The channel parameters are determined stochastically, based on statistical distributions extracted from channel measurements. The distributions are defined for, e.g. delay spread, delay values, angle spread, shadow fading, and cross-polarization ratio. For each channel segment the channel parameters are calculated from the distributions. Specific channel realizations are generated by summing contributions of rays with specific channel parameters like delay, power, angle-of-arrival and angle-of-departure. Different scenarios are modeled by using the same approach, but different parameters.

Note that the use of GSCM permits to have a fair trade off between computation time and complexity cost of the channel simulation and realism. Indeed, when designing a wireless transmission system, it is useful to evaluate its performance over at least a minimum number of channel realizations. The high computational complexity of deterministic modelling prohibits the intensive link or system level simulation required during system design. That is why GSCM modelling is the most used approach in practice.

The QuaDRiGa approach can be understood as a "statistical ray-tracing model". Unlike the classical ray tracing approach, it does not use an exact geometric representation of the environment but distributes the positions of the scattering clusters (the sources of indirect signals such as buildings or trees) randomly. A simplified overview of the model is depicted in Figure 1. For each path, the model derives the angle of departure (the angle between the transmitter and the scattering cluster), the angle of arrival (the angle between the receiver and the scattering cluster) and the total path length which results in a delay $\tau$ of the signal. For the sake of simplicity, only two paths are shown in the figure. 


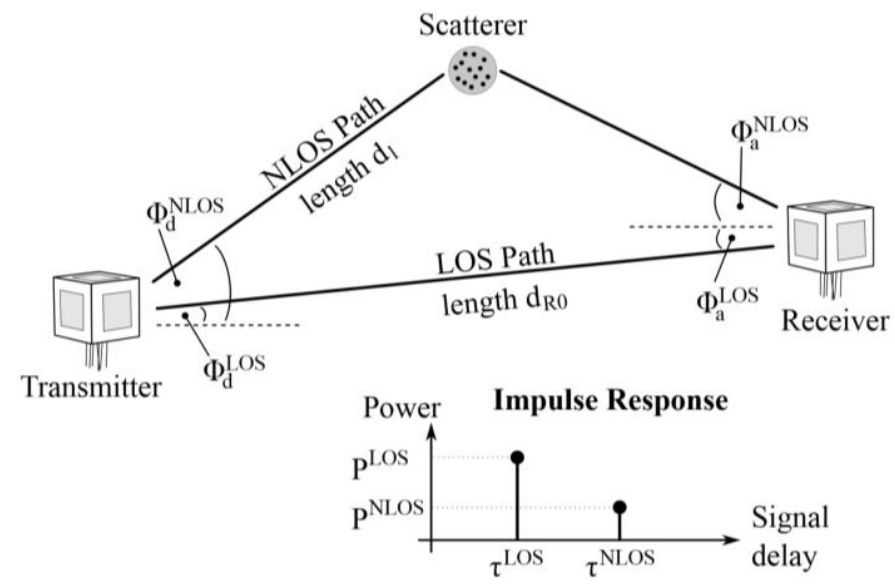

Figure 1 - Simplified overview of the modelling approach in QuaDRiGa - source [16]

In Figure 2, a snapshot of one particular scenario is proposed. As it can be seen, in addition to the LOS path linking the transmitter and the receiver (black solid line), QuaDRiGa generates a set of randomly distributed clusters illustrated by their First and Last Bounce Scatterers.

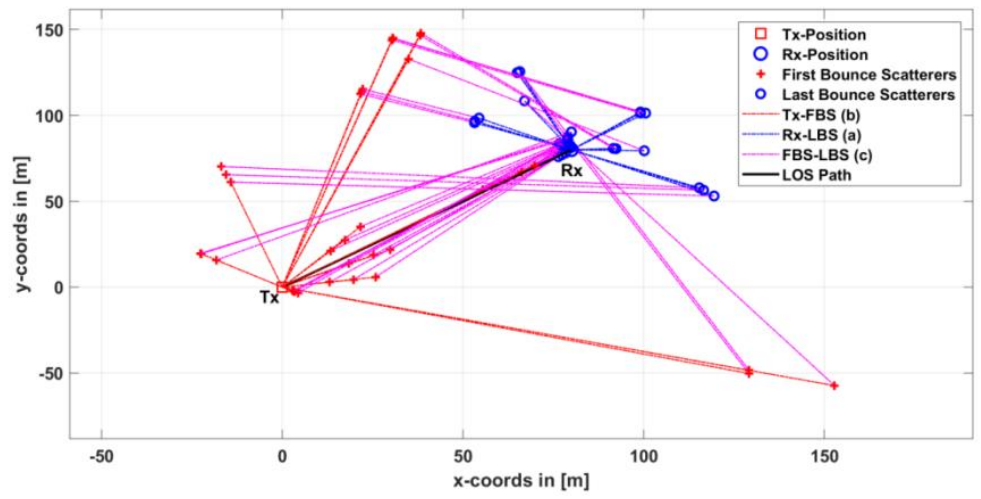

Figure 2 - clusters randomly distributes - illustration

In Figure 3, an example of the path power and delays generated by QuaDRiGa is presented. The trajectory considered here is a $50 \mathrm{~m}$-radius-circle. The scenario is a Non-Line-Of-Sight (NLOS) Urban Micro Cell scenario; it models typical terrestrial pico-base stations deployed below rooftop in densely populated urban areas covering carrier frequencies from $500 \mathrm{MHz}$ to $100 \mathrm{GHz}$. The chosen carrier frequency is $2 \mathrm{GHz}$.
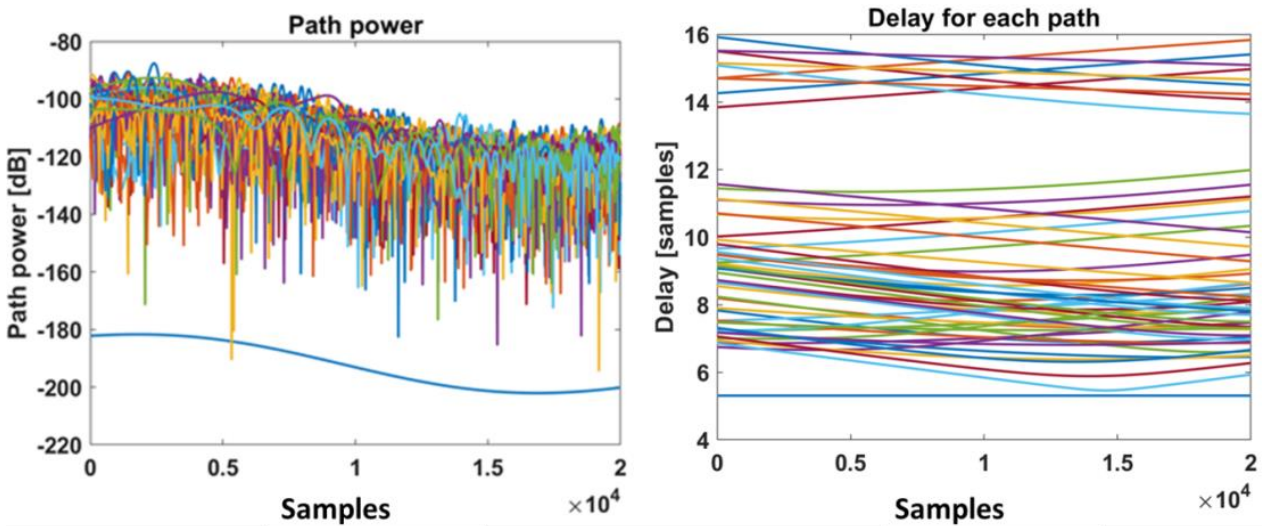

Figure 3 - "3GPP_38.901_UMi_NLOS" scenario - carrier frequency set to 2GHz - trajectory circular 50m radius - QuaDRiGa 
The QuaDRiGa propagation channel model adopted from now on is given in (2).

$$
\alpha^{k}(t, \tau)=\sum_{l=0}^{L-1} \alpha_{l}^{k}(t, \tau)=\sum_{l=0}^{L-1} \alpha_{l}^{k}(t) \cdot \delta\left(\tau-\tau_{l}(t)\right), 0 \leq t \leq T_{\text {OFDM }}
$$

Where $\alpha_{l}^{k}$ is the channel complex amplitude for the $k^{\text {th }}$ OFDM symbol and for the $l^{\text {th }}$ path, and $T_{O F D M}$ is the OFDM symbol duration.

In the article, the scenario selected is the QuaDRiGa 3GPP_38.901_UMi_LOS scenario it models typical terrestrial picobase stations deployed below rooftop in densely populated urban areas covering carrier frequencies from $500 \mathrm{MHz}$ to $100 \mathrm{GHz}$. It fulfills both the frequency and constrained environment requirements.

\section{2 - SCHUN for GNSS systems}

Regarding GNSS systems, several studies have been conducted in the community to design a realistic propagation channel. As for $5 \mathrm{G}$ propagation channel, a trade-off has been taken in order to select a realistic and fast computing propagation channel. The selected one is SCHUN, [8], a mobile satellite channel simulator based on a hybrid approach satisfying accuracy and fast computation requirements.

SCHUN stands for Simplified Channel for Urban Navigation; it is a hybrid physical-statistical Land Mobile Satellite propagation channel. The complete description of the model can be found in [17]. The macro architecture of SCHUN is provided in Figure 4. Basically, SCHUN generates the environment by using a random virtual city approach; this is the statistic aspect of the model. Then, based on this generated environment, it models the interactions between impinging signals and the environment by using simple Electromagnetic (EM) interaction models; this is the deterministic aspect of the model.

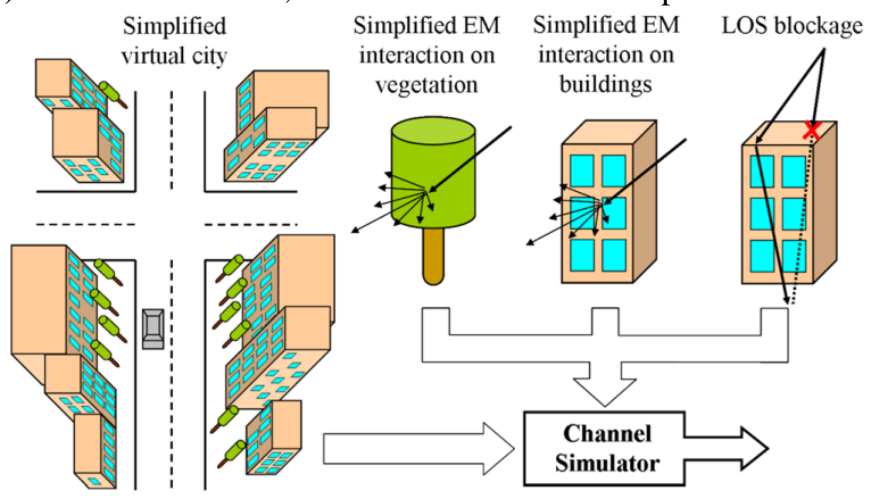

Figure 4 - SCHUN macro architecture - source [17]

The SCHUN propagation channel model adopted from now on is given in (3).

$$
\alpha(t, \tau)=\sum_{l=0}^{N} \gamma_{l}(t) e^{-j \Phi_{l}(t)} \delta\left(\tau-\tau_{l}(t)\right)
$$

Where:

$\circ \quad t$ is the time variable

- $\tau$ is the convolution variable

- $\alpha(t, \tau)$ is the time delay impulse response of the channel

- $\quad N$ is the number of multipath

- $\gamma_{l}$ is the ray amplitude

- $\Phi_{l}$ is the phase of the ray including delay and reflection shifts

$\circ \tau_{l}$ is the ray delay

\section{GENERATION OF HIGHLY REALISTIC CORRELATOR OUTPUTS}

One of the objectives of the paper is to realistically characterize the $5 \mathrm{G}$ and GNSS pseudo ranges measurements. In order to obtain the pseudo ranges measurements to be statistically characterize, the methodology adopted in this document consists in:

- Generations the pseudo range measurements through simulations

- Directly generating the correlator outputs associated to the reception of 5G and GNSS signals rather than the complete signals before correlation (DS-SS signals for GNSS and OFDM signals for 5G)

The main reason for using simulations is the actual lack of a true 5G network deployment as well as an easy control of the user trajectory with respect to the emitter (satellite or base station). In fact, note that to obtain a complete and significant pseudo range 
statistical characterization, a very high number of different trajectories must be analyzed. Therefore, being able to simulate all these trajectories was considered to be the most practical solution. The main reason for directly generating the correlator outputs instead of the complete signal was to speed up a very time-consuming process: a lot of trajectories and a lot of runs of the same trajectory should be generated. Moreover, note that the correlator outputs are at the basis of the ranging modules envisioned for GNSS and $5 \mathrm{G}$; which are responsible for the generation of the pseudo range measurements. Therefore, the correlator output mathematical models of both GNSS and 5G systems have to be perfectly defined.

In the following, the analyses are made on correlator outputs. Indeed, GNSS and 5G signal processing modules are based on correlator outputs. Those correlator outputs are at the basis of all the synchronization process. Thus the mathematical models of these correlator outputs must be perfectly defined.

First the 5G correlator output mathematical model is derived; second the GNSS correlator outputs are reminded.

\section{1 - 5G correlator output mathematical model}

Previous efforts have been made to provide the mathematical model of the $5 \mathrm{G}$ received signal at the different receiver signal processing stages, see [18], [19]. However, these models are derived assuming a propagation channel constant over the duration of the OFDM symbol duration. An analysis of QuaDRiGa has proved that the propagation channel is evolving over the duration of the OFDM symbol, [12]. Thus classical models have to be refined.

5G systems, according to [11], will use Orthogonal Frequency Division Multiplexing (OFDM) signals. Assuming a

sampling period $T_{S}=\frac{1}{N_{F F T} \Delta f}$, where $N_{F F T}$ is the FFT size and $\Delta f$ the subcarrier spacing, the discrete model of the transmitted signal for the OFDM symbol's duration is defined as:

$$
s^{k}[n]=\sum_{q=0}^{N_{F F T}-1} d_{q}^{k} e^{\frac{i 2 \pi p n}{N_{F F T}}}-N_{C P} \leq n \leq N_{F F T}-1
$$

Assuming the propagation channel model,(2), the discrete received signal model at the Radio Frequency (RF) front-end output over the duration of one OFDM symbol is thus defined as (5).

$$
r_{n}^{k}=\sum_{l=0}^{L-1} \alpha_{l}^{k}(n) \sum_{q=0}^{N_{F F T}-1} d_{q}^{k} e^{\frac{i 2 \pi q\left(n-\tilde{\tau}_{l}(n)\right)}{N_{F F T}}}+w_{n}^{k}
$$

Where $w_{n}^{k}=w_{n_{I}}^{k}+j w_{n_{Q}}^{k}$, with $w_{n_{I}}^{k}$ and $w_{n_{Q}}^{k}$ two independent zero-mean Gaussian variables with $N\left(0, \frac{\sigma_{\text {noise }}^{2}}{2}\right)$, $\sigma_{\text {noise }}^{2}$ is the RF front-end noise power. In order to derive the mathematical models of the demodulated symbols and of the correlator outputs, in the next sections, the focus is on the useful part of the signal (without the noise term $w_{n}^{k}$ in (5)). In fact the noise contribution will be added, as a noise correlator output term, in the final expression of the correlator outputs mathematical model in (9).

As a reminder, the principle of the correlation is to correlate the incoming demodulated signal with a local replica of the signal. In order to understand the generated local replica, a brief description of the OFDM signal architecture is reminded, details can be found in [11].

In an OFDM modulation $\mathrm{N}$ complex data symbols are transmitted over $\mathrm{N}$ orthogonal narrowband subcarriers; thus, defining a time/frequency resource grid of symbols. The time step is the OFDM symbol duration, the frequency step corresponds to the subcarrier spacing. A resource grid is composed of 2 types of symbols: data and pilots. The data symbols corresponds the useful information for the user - a-priori unknown. The pilot symbols are known symbols in terms of time/frequency localization and value. Due to the knowledge of their location, pilot symbols present interesting correlation properties; thus the mathematical model presented in this paper is based on correlation operations over reference pilot signals, a method already used in [20], [8].

The general correlation formula is presented in (7):

$$
R\left(\varepsilon_{\tau}\right)=\frac{1}{N_{P}} \sum_{q^{\prime} \in P} \hat{d}_{q^{\prime}}^{k} \cdot F L R_{q^{\prime}}^{k *}[\hat{\tau}]
$$

Where $F L R_{q^{\prime}}^{k},[\hat{\tau}]=\operatorname{FFT}\left(L R^{k}[\hat{\tau}]\right)_{q^{\prime}}=p_{q^{\prime}}^{k} e^{-\frac{2 i \pi q^{\prime}}{N_{F F T}} \hat{\tau}}$ is the local replica with $p_{q^{\prime}}^{k}$ the pilot symbol localized in the $q^{\prime t h}$ subcarrier on the $k^{\text {th }}$ OFDM symbol, $N_{p}$ is the number of pilot symbols, $\hat{d}_{q^{\prime}}^{k}$ is the demodulated symbols and $P$ is the set of pilots.

$5 \mathrm{G}$ systems flexibility forbids the definition of a complete deterministic resource grid. Only one set of pilots is predictable and can thus be used: 4-OFDM-symbol-long Synchronization Signal Physical Broadcast CHannel (SSPBCH). The detection and decoding of this block allow the user to achieve downlink synchronization in time/frequency domain and to obtain $5 \mathrm{G}$ signal characteristics such as the cell identity or the bandwidth; information needed to perform the communication with the network. In the following, the focus is put on the 2nd and 4th symbols which are composed of 1 Demodulation Reference Signal (DMRS) 
every 4 subcarriers over 240 subcarriers, details in [20], the time/frequency allocation of a SSPBCH block is provided in [21] and illustrated in Figure 5.

In the following of the study, the pilot architecture used for the correlation corresponds to the second or forth symbol of the SSPBCH block. Thus, in order to have a generic formula, the set of pilots $P$ in (6) can be defined as: $P=\gamma q^{\prime}+\beta$. Where $\gamma$ is the period of repetition of the pilots in the OFDM symbol, $q^{\prime} \in\left[0 \ldots N_{P}-1\right]$ and $\beta$ is the subcarrier index of the first pilot in the symbol, see Figure 5.

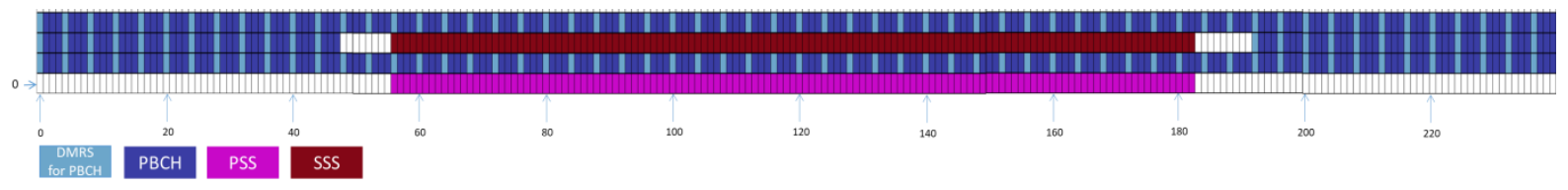

Figure 5 - Frequency domain allocation

In order to derive the mathematical models of the correlator outputs, the focus is on the useful part of the signal (without the noise term $w_{n}^{k}$ in (5)). In fact the noise contribution will be added, as a noise term, in the final expression of the correlator outputs mathematical model in (10).

$$
\begin{gathered}
R\left(\varepsilon_{\tau}\right)=\frac{1}{N_{P}} \sum_{q^{\prime} \in P}\left(\hat{d}_{q_{\text {useful }}^{\prime}}^{k}+\hat{d}_{q_{\text {interf }}^{\prime}}^{k}\right) p_{q^{\prime}}^{k *} e^{\frac{2 i \pi \hat{\tau} q^{\prime}}{N_{F F T}}} \\
R\left(\varepsilon_{\tau}\right)=R_{\text {useful }^{\prime}}\left(\varepsilon_{\tau}\right)+R_{\text {interf }}^{\text {pilot }}\left(\varepsilon_{\tau}\right)+R_{\text {interf }}^{\text {data }}\left(\varepsilon_{\tau}\right)
\end{gathered}
$$

Where $R_{\text {useful }}\left(\varepsilon_{\tau}\right)=\sum_{l=0}^{L-1} \frac{A_{l}^{k}(0)}{N_{F F T}} R_{l}\left(\varepsilon_{\tau_{l}}\right)$ and $A_{l}^{k}(0)=\sum_{n=0}^{N_{F F T}-1} \alpha_{l}^{k}(n)$ represents the evolution of the propagation channel over one symbol for the $l^{\text {th }}$ path, $R_{l}\left(\varepsilon_{\tau_{l}}\right)$ is provided in (9), $R_{\text {interf }}$ data is an interference term due to the data present in the OFDM symbol, $R_{\text {interf }}^{\text {pilot }}$ is an interference term due to the pilots; their expression are provided in (9). These last two interference terms are Inter Carrier Interference (ICI); the use of the OFDM modulation should avoid these kinds of ICI however, here, they came from the evolution of the propagation channel.

$$
\begin{aligned}
& R_{l}\left(\varepsilon_{\tau_{l}}\right)=\left\{\begin{array}{cl}
\frac{1}{N_{P}} e^{\frac{i 2 \pi \beta \varepsilon_{\tau_{l}}}{N_{F F T}} \frac{\sin \left(\frac{\pi \gamma \varepsilon_{\tau_{l}} N_{P}}{N_{F F T}}\right)}{\sin \left(\frac{\pi \gamma \varepsilon_{\tau_{l}}}{N_{F F T}}\right)} e^{\frac{i \pi \gamma\left(N_{P}-1\right) \varepsilon_{\tau_{l}}}{N_{F F T}}}} & \varepsilon_{\tau_{l}} \neq 0 \\
1 & \varepsilon_{\tau_{l}}=0
\end{array}\right.
\end{aligned}
$$

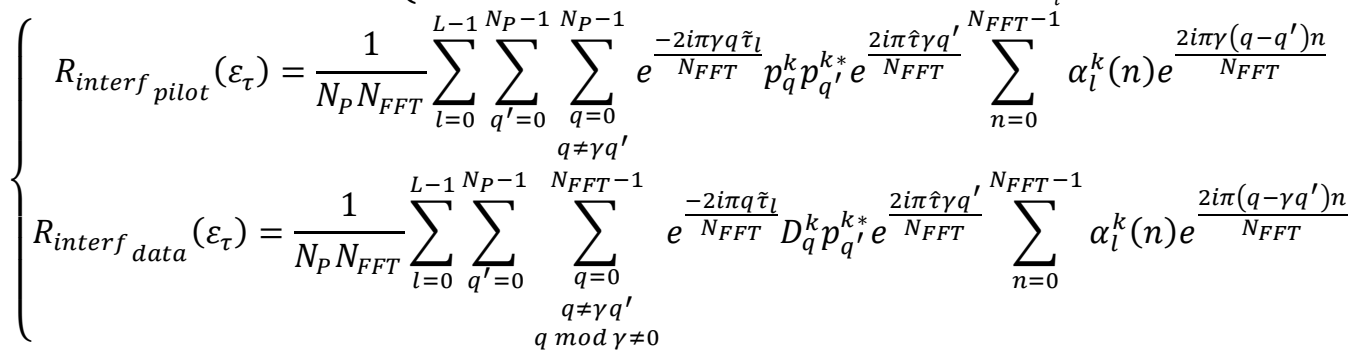

In [12], studies have proved that in targeted scenario, the correlator output mathematical models simulated can be limited to (9), $n$ is the noise contribution which theoretical development can be found in [12].

\section{2 - GNSS correlator output mathematical model}

$$
R\left(\varepsilon_{\tau}\right)=R_{\text {useful }}\left(\varepsilon_{\tau}\right)+n\left(\varepsilon_{\tau}\right)
$$

Regarding GNSS systems, correlator outputs mathematical models are well known and derived in [11]. In this section a short derivation of the models applied to the multipath environment are provided.

Assuming the SCHUN propagation channel model (3), the GNSS In-Phase and In-Quadrature correlator output mathematical models implemented are, for the Prompt way considering the previous propagation channel model: 
Where:

$$
\left\{\begin{array}{l}
I_{P}(k)=\sum_{l=0}^{N_{\text {multi }}-1} \frac{\gamma_{l_{k}} A d_{k}}{2} K_{c, c_{l}}\left(\varepsilon_{\tau_{l}}\right) \cos \left(\pi \varepsilon_{f_{l}} T_{I}+\varepsilon_{\Phi_{l}}\right) \operatorname{sinc}\left(\pi \varepsilon_{f_{l}} T_{I}\right) \\
Q_{P}(k)=\sum_{l=0}^{N_{\text {multi }}-1} \frac{\gamma_{l_{k}} A d_{k}}{2} K_{c, c_{l}}\left(\varepsilon_{\tau_{l}}\right) \sin \left(\pi \varepsilon_{f_{l}} T_{I}+\varepsilon_{\Phi_{l}}\right) \operatorname{sinc}\left(\pi \varepsilon_{f_{l}} T_{I}\right)
\end{array}\right.
$$

- $\quad \varepsilon_{\tau_{l}}=\tau_{l_{k}}-\hat{\tau}_{k}$ the delay estimation error

- $\quad \tau_{l_{k}}$ the $l$ path delay at instant $k$

- $\quad \hat{\tau}_{k}$ the estimated delay from the DLL

- $\quad \varepsilon_{f_{l}}=\Delta f_{d_{l_{k}}}-\widehat{\Delta f_{d}}$, it the Doppler estimation error

- $\quad \Delta f_{d_{l_{k}}}$ the $l$ path doppler at instant $k$

- $\widehat{\Delta f_{d}}$ the estimated doppler

- $\quad \varepsilon_{\Phi_{l}}=\Phi_{l_{0}}-\widehat{\varphi}_{k}$ is the phase estimation error at the beginning of the interval

- $\quad \Phi_{l_{0}}$ the $l$ path phase at the beginning of the interval at instant $k$

- $\quad \widehat{\varphi}_{k}$ the estimated phase at the beginning of the interval

- $\quad K_{c, c_{l}}=T_{I} \int_{\hat{\tau}+k T_{I}}^{\hat{\imath}+(k+1) T_{I}} c\left(u-\tau_{l_{k}}\right) c_{l}(u-\hat{\tau}) d u$ is the autocorrelation of the code

In this section the correlator output mathematical models for GNSS and 5G systems have been derived. These correlator outputs are required since they are at the basis of the ranging module which provides the pseudo range measurements.

\section{CHARACTERIZATION OF PSEUDO RANGE ERROR DISTRIBUTIONS}

The propagation channels SCHUN and QuaDRiGa and the thermal noise introduce errors on the pseudo range measurements. Kalman Filters assume that these errors follow Gaussian distribution; an assumption that may be too restrictive in urban environments and may degrades the positioning performances by over-bounding the measurement error. Monte Carlo simulations will permit to characterize accurately in terms of mean, variance and probability density functions (PDF); then these characterizations will be used in the navigation filters. The objectives is, by accurately characterize the distribution to improve the navigation solution.

First the methods developed for this study to over bound and to fit the distributions are presented. Then the obtained characterization for QuaDRiGa and SCHUN are presented. The characterization is made in function of the $C / N_{0}$.

\section{1 - Development of the over bounding and fitting method}

Two methods have been designed to characterize the errors: a fitting method and an over-bounding method. There is no obvious reason to choose one or the other, both will be tested in the navigation filters in order to choose the more appropriate for our application. The presentation of the methods is made through an example. First the running example is presented, then the over bounding method is presented and finally the fitting method is presented. The error distribution is over bounded or fitted by a Gaussian distribution.

\subsubsection{Running example presentation}

In Figure 6, the pseudo ranges considered for the example are presented. The pseudo ranges are generated from a $25 \mathrm{~m}$ radius circle trajectory; the scenario selected is the QuaDRiGa 3GPP_38.901_UMi_LOS scenario it models typical terrestrial picobase stations deployed below rooftop in densely populated urban areas covering carrier frequencies from $500 \mathrm{MHz}$ to $100 \mathrm{GHz}$. Regarding the receiver processing, the OFDM numerology considered consists in a subcarrier spacing of $15 \mathrm{kHz}$ and a carrier frequency of $2 \mathrm{GHz}$. The correlator outputs generated are used by the ranging module; the DLL considered uses an Early Minus Late Power (EMLP) discriminator, the loop bandwidth is set to $10 \mathrm{~Hz}$ and the correlator spacing is set to 4 samples. A second order DLL is considered. A complete description of the ranging module is presented in [12]. 


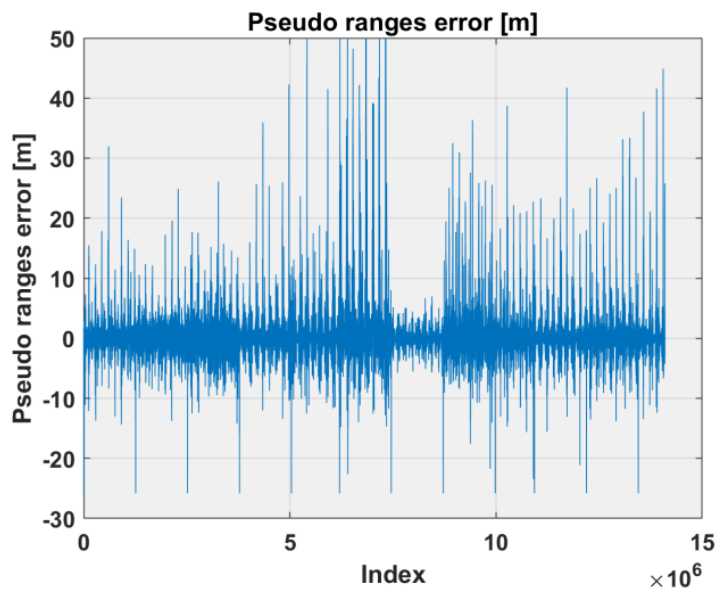

Figure 6 - Pseudo range errors

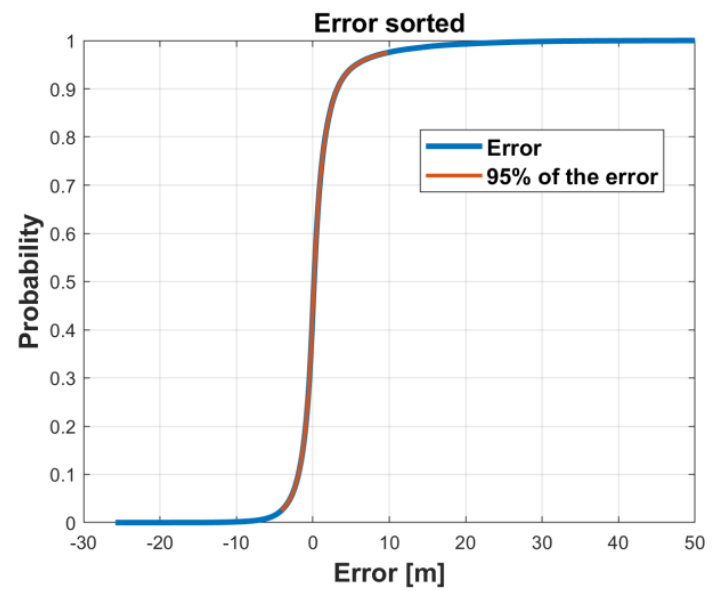

Figure 7 - Selection of the errors to be over bounded or fitted

In Figure 7 (blue curve), the pseudo range errors from Figure 6 are sorted to obtain the Cumulative Density Function (CDF). The fitting and the over bounding are made thanks to the CDF.

\subsubsection{Characterization parameters}

In order to fit and to over-bound this distribution, 3 confidence bounds are tested: $95 \%, 99 \%$ and $99.9 \%$. In order to characterize the distribution at $X \%$, the errors that are kept correspond to the CDF errors comprised in the interval $\left[\left(\frac{100-X}{2}\right):\left(100-\frac{100-X}{2}\right)\right]$. In Figure 7 , in red are presented the error considered to find the $95 \%$ fitting/over-bounding distributions; it corresponds to the errors in the interval [2.5\%:97.5\%]. The rest of the section will focus on the $95 \%$ characterization.

This distribution will be characterize by three Gaussian distributions, $N(\mu, \sigma)$. These 3 distributions differs by the considered value of the mean $\mu$ :

- $\mu=0$

- $\quad \mu=$ mean, where mean corresponds to the mean value of the considered errors, the mean of the red curve in Figure 7

\subsubsection{Over bounding method}

$\mu=\max$, where index corresponds to the index of the maximum of the histogram of the considered errors.

The over-bounding method consists in generating a Gaussian CDF initialized with a high variance value and to decrease the variance until the over bounding CFD envelop the CDF of the error to be over bounded. Figure 8 illustrates the process.

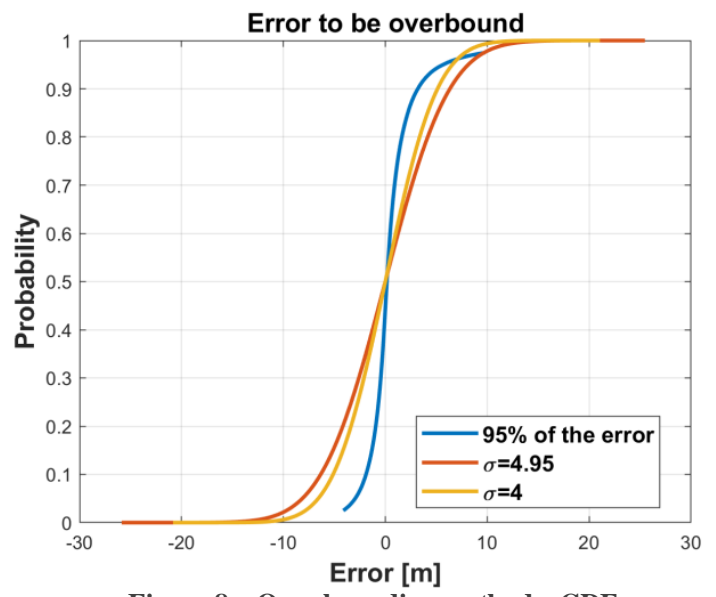

Figure 8 - Over bounding method - CDF

In Figure 8, the CDF to be over bounded is the blue curve; the errors are over bounded by a zero mean Gaussian distribution. The red curve fulfils the over bounding conditions since the red curve is always at the right of the blue curve for 
positive error and at the left of the blue curve for the negative errors. On the contrary, the yellow curve does not overlap entirely the positive errors.

\subsubsection{Fitting method}

The fitting method consist in generating a Gaussian CDF initialized with a small variance value and to increase the variance while the norm of the error over the $\mathrm{X}$ axis between the fitting CFD and the CDF of the error to be fitted is not minimal. Figure 9 and Figure 10 illustrates the process.

$$
\min _{\sigma}|| C D F_{\text {error }}-C D F_{\text {fitting }}(\sigma)||
$$

Where $C D F_{x}$ represents the $C D F$ vector of source $x$.

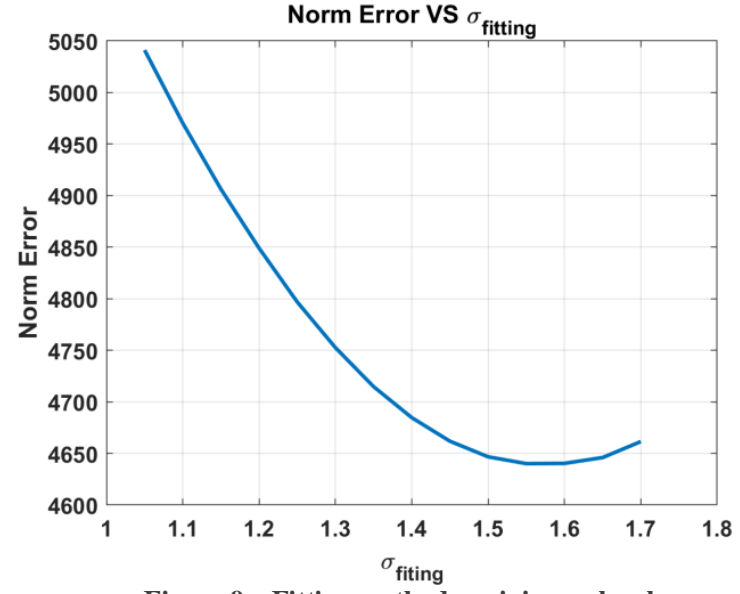

Figure 9 - Fitting method - minimum local

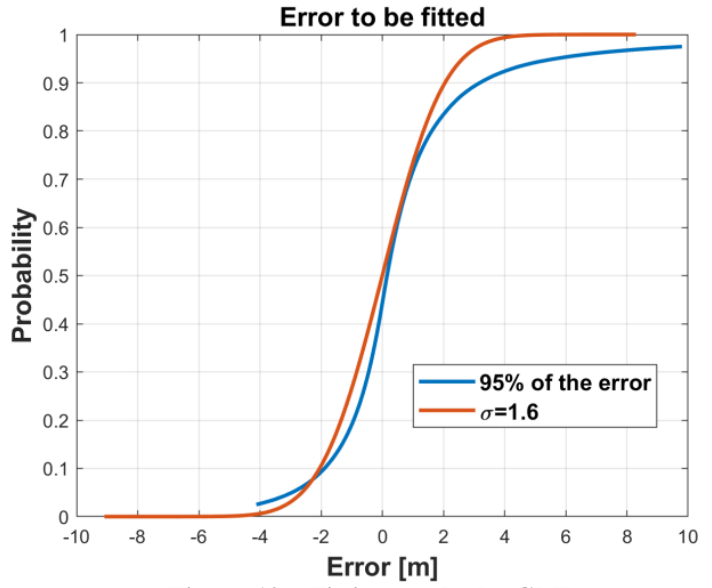

Figure 10 - Fitting method - CDF

As it can be observed from Figure 9, the norm of the error is decreasing as the value of the variance increases until it reaches a minimum local which corresponds to the fitting distribution illustrated in Figure 10.

\subsubsection{Methods comparison}

In Figure 11 and Figure 12, the CDF and PFD of the fitting and over-bounding method are provided. As it can be seen, both methods provide very different results. Basically, the fitting method permits to correctly characterize the small errors, the center of the distribution. On the contrary, the over bounding method will allow to cover the tails of the distribution.

In this study, both methods are tested and compared in terms of positioning performances. Recommendations will be made according to the performances obtained.

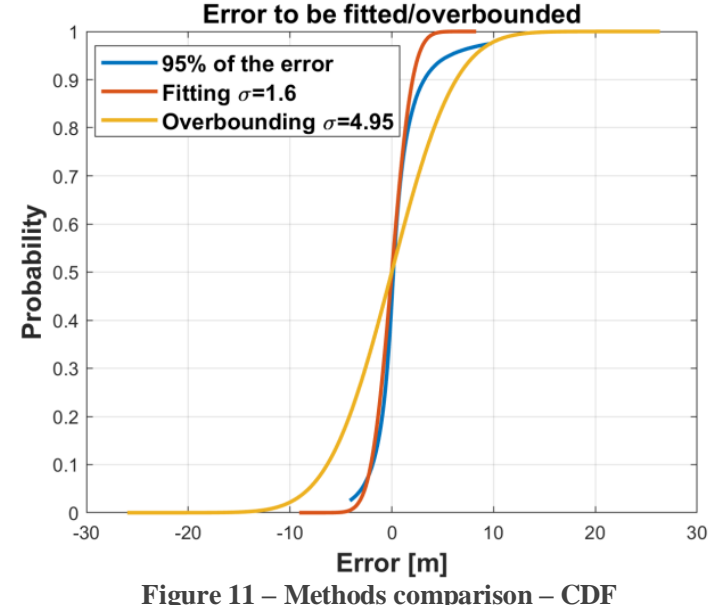

Figure 11 - Methods comparison - CDF

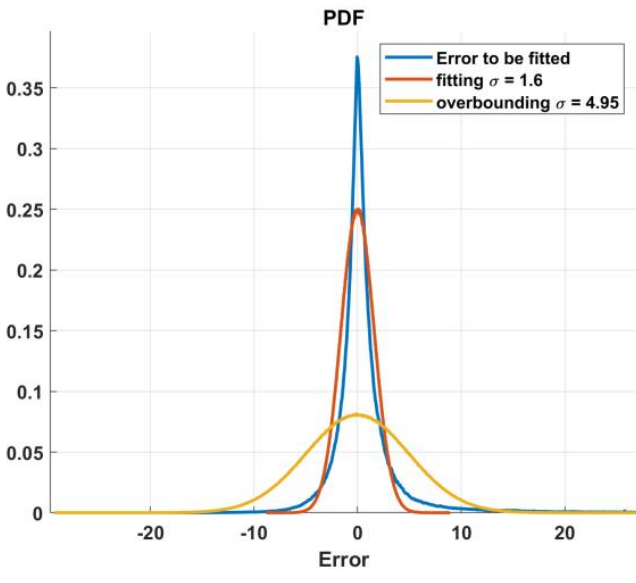

Figure 12 - Method comparison - PDF

\section{2 - QuaDRiGa characterization}

In this section the pseudo range error characterization for $5 \mathrm{G}$ signals is provided. The errors on the pseudo ranges are due to the thermal noise and the QuaDRiGa propagation channel. Thus, first the scenario generated are presented; second the over bounding and fitting results are provided. 


\subsubsection{Generated scenario}

The scenario design of the propagation channel to be simulated by QuaDRiGa is made through the selection of 3 parameters: high-level scenario, receiver's trajectory and signal characteristics.

Regarding the high-level scenarios, the configuration used is referred as 3GPP_38.901_UMi_LOS. The configuration models typical terrestrial pico-base stations deployed below rooftop in densely populated urban areas covering carrier frequencies from $500 \mathrm{MHz}$ to $100 \mathrm{GHz}$. The interest in choosing this high-level scenario is twofold. First, it follows the 3GPP standard on 5G propagation channel: TS.38.901, [22], especially regarding the frequency range. Second, it models Urban Micro-Cell environment which fits with the problematic (constrained environment). The LOS scenario is a favorable case meaning that the LOS signal has higher power than multipath.

Regarding the trajectories, since the scenario selected simulates pico-base stations which have typically a 4-to-200 mradius-size, 12 circular trajectories have been studied, that lies in 10-to-250 m-radius-size. The radiuses are a bit larger than the maximum pico-cell radius in order to increase the number of points at the edge. Indeed, the characterization is made with respect to the $\mathrm{C} / \mathrm{N} 0$ and the objective is to correctly characterize the pseudo range error even at low $\mathrm{C} / \mathrm{N} 0$ (when at the limit of the pico-cell).

The numerology of the OFDM signal is a $15 \mathrm{kHz}$ subcarrier spacing and a $2 \mathrm{GHz}$ carrier frequency. Regarding the receiver characteristics, a second order DLL is used; two loop bandwidths are considered: $1 \mathrm{~Hz}$ and $10 \mathrm{~Hz}$ and the correlator spacing is set to 4 samples.

Finally, the last important point is the definition of the input SNR and the estimated $C / N_{0}$; indeed, remind that the driven idea remains to be as realistic as possible. It is assumed that the useful transmitted power at the base station output is set to $C=0 \mathrm{dBm}=-30 \mathrm{dBw}$. Note that according to $3 \mathrm{GPP}$ standards, the maximum transmitted power is $C_{\max }=23 \mathrm{dBm}$, in order to simulate a less ideal or more realistic case the transmitted power has been decreased to $C=0 \mathrm{dBm}$. The noise power is defined using the classical GNSS receiver noise value $N_{0}=-200 \mathrm{dBw}$ and the bandwidth $B=\Delta f \cdot N_{F F T}$ where $N_{F F T}$ is the FFT window size and $\Delta f$ is the subcarrier spacing of the OFDM signal. The SNR at the demodulation input and without considering the propagation channel is thus $S N R_{\text {demodulation }}=\frac{C}{N_{0} B} \cong 95 \mathrm{~dB}$. In order to estimate the C/N0 at the correlator output, the estimator considered is derived in [23] and it is based on the comparison of total signal-plus-noise power in two different bandwidths can be used to determine signal-plus-noise ratio.

\subsubsection{Results}

The characterization is made with respect to the estimated $C / N_{0}$. Results for the fitting characterization are provided in Figure 13 for the loop bandwidths of $1 \mathrm{~Hz}$ and $10 \mathrm{~Hz}$ respectively. Results for the over bounding characterization are provided in Figure 14 for the loop bandwidths of $1 \mathrm{~Hz}$ and $10 \mathrm{~Hz}$ respectively.

From these figures it can be seen that the differences between the 3 Gaussian distributions, $N(0, \sigma), N($ mean,$\sigma)$ and $N(\max , \sigma)$ is negligible. More specifically, the fitting and over bounding distributions $N(0, \sigma)$ and $N(\max , \sigma)$ are barely identical since the maximum of the PDF distribution of the errors is almost $0(\max \cong 0)$. The difference with the $N($ mean, $\sigma)$ is slightly larger especially at low $\mathrm{C} / \mathrm{N} 0$ where the value of the mean is increasing.

Second, the variance of the over bounding and fitting distributions is increasing as the C/N0 decreases. This is quite obvious; the pseudo range quality gets lower with the C/NO.

Finally the variances seem to reach a threshold at high C/NO. Here again the explanation is quite easy to understand; as the $\mathrm{C} / \mathrm{N} 0$ gets higher, the propagation channel impact decreases and only the thermal noise remains since we have considered a LOS scenario. 

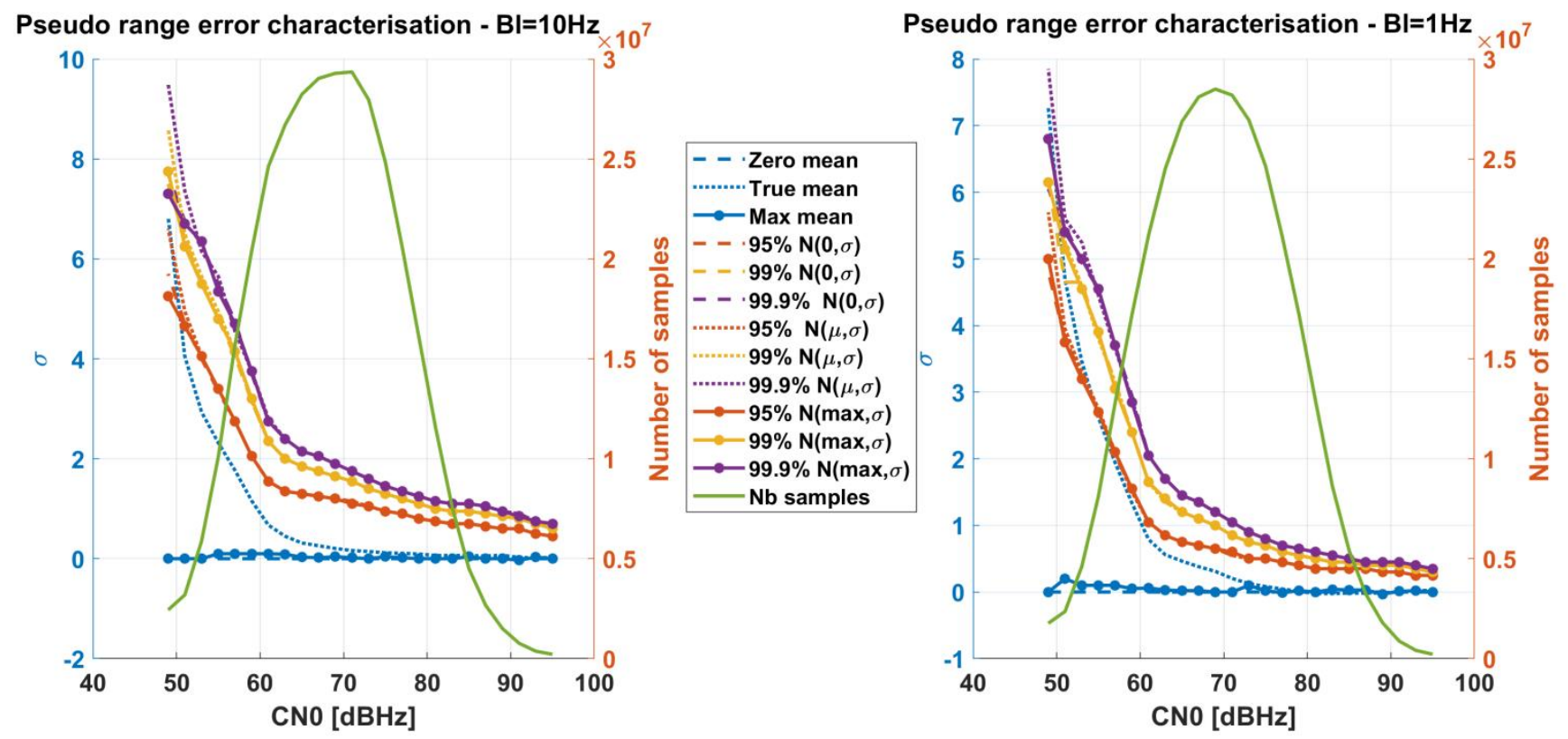

Figure 13 - 5G characterization - Fitting
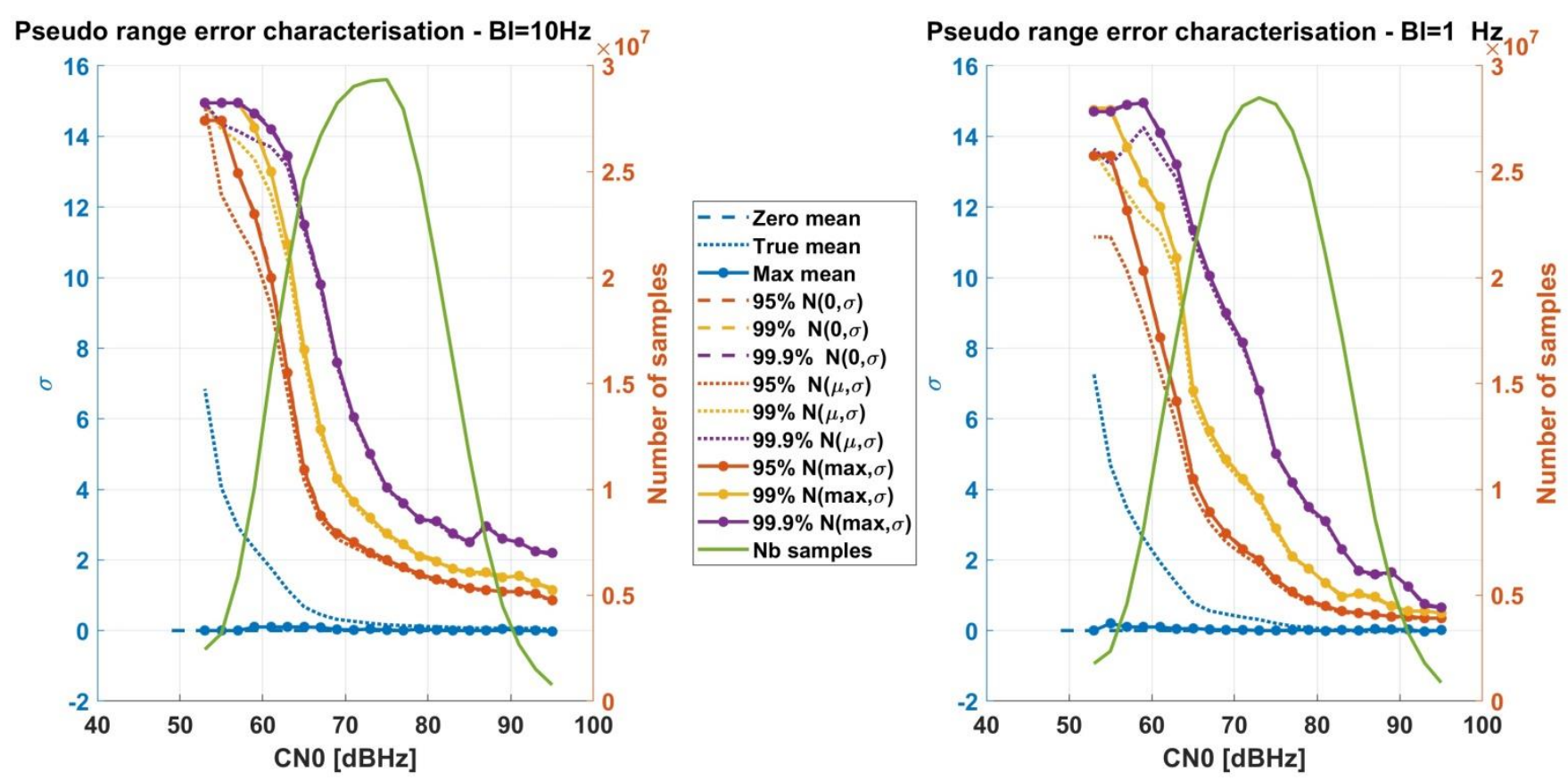

Figure 14 - 5G characterization - Overbounding

In order to compare the behavior of the over bounding results and the fitting results, Figure 15 is provided. Basically, the over bounding variances are always larger than the fitting variances. The difference increases as the C/N0 decreases. 

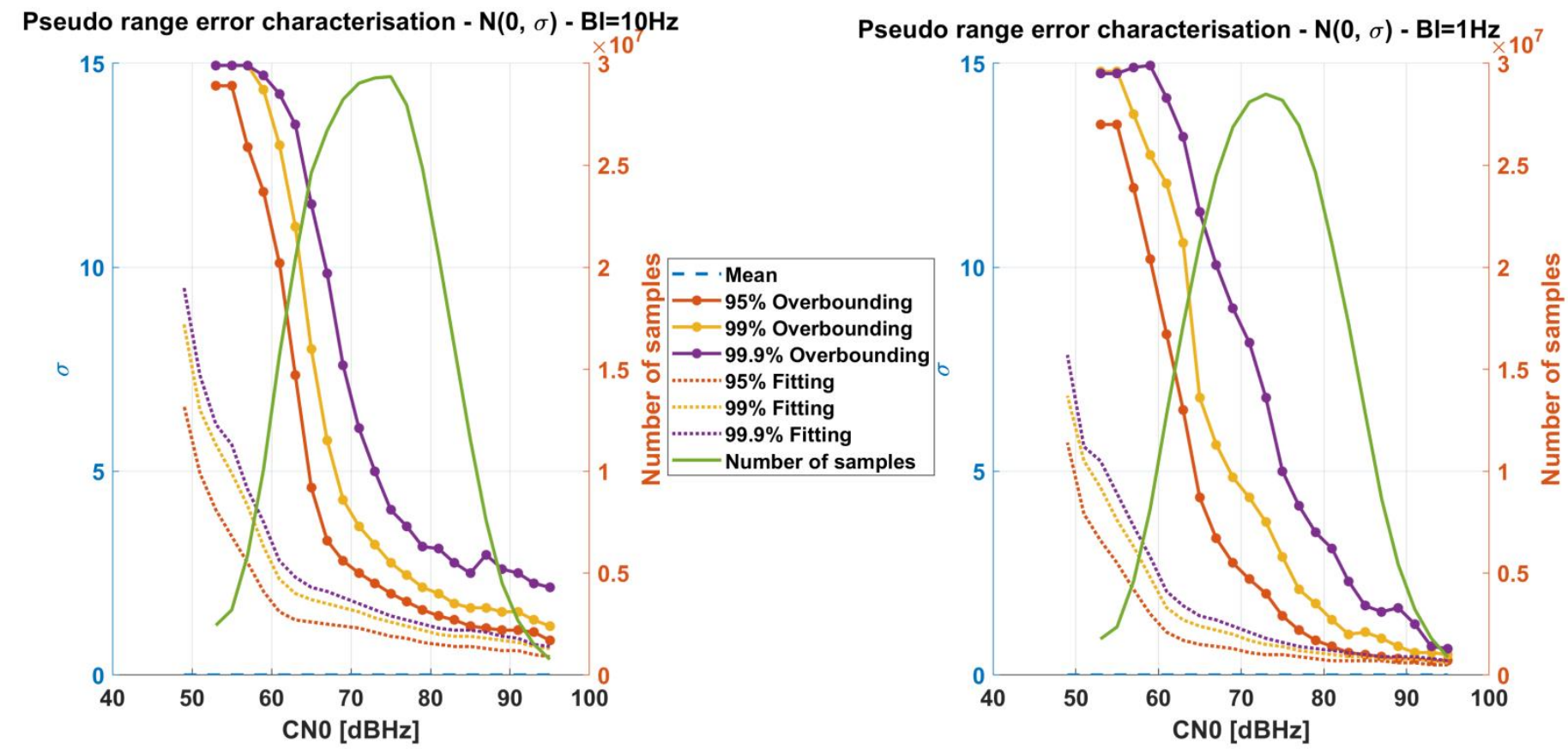

Figure 15 - 5G characterization $-N(0, \sigma)$

In Table 1, the $\sigma$-values obtained for the $N(0, \sigma)$ and for a $10 \mathrm{~Hz}$ loop bandwidth are provided. They are used in the simulations of the next sections. More specifically, in the simulation the $\sigma$-values used corresponds to the $99.9 \%$-columns.

Table 1 - Characterization values obtained for a $N(0, \sigma)$ for a $10 \mathrm{~Hz}$ loop bandwidth

\begin{tabular}{|c|c|c|c|c|c|c|}
\hline & \multicolumn{9}{|c|}{$\sigma$} & \multicolumn{3}{c|}{ Fitting } \\
\hline & \multicolumn{3}{|c|}{ Overbounding } & \multicolumn{3}{|c|}{} \\
\hline$C / N_{0}[\mathrm{dBHz}]$ & $95 \%$ & $99 \%$ & $99,90 \%$ & $95 \%$ & $99 \%$ & $99,90 \%$ \\
\hline 95 & 0.85 & 1.2 & 2.15 & 0.45 & 0.6 & 0.7 \\
\hline 93 & 1.05 & 1.35 & 2.25 & 0.5 & 0.7 & 0.75 \\
\hline 91 & 1.1 & 1.55 & 2.5 & 0.6 & 0.8 & 0.9 \\
\hline 89 & 1.1 & 1.55 & 2.6 & 0.6 & 0.85 & 0.95 \\
\hline 87 & 1.15 & 1.65 & 2.95 & 0.65 & 0.9 & 1.05 \\
\hline 85 & 1.2 & 1.65 & 2.5 & 0.7 & 0.95 & 1.1 \\
\hline 83 & 1.35 & 1.75 & 2.75 & 0.7 & 0.95 & 1.1 \\
\hline 81 & 1.45 & 2.0 & 3.1 & 0.75 & 1.0 & 1.15 \\
\hline 79 & 1.6 & 2.15 & 3.15 & 0.8 & 1.1 & 1.25 \\
\hline 77 & 1.8 & 2.45 & 3.65 & 0.9 & 1.2 & 1.35 \\
\hline 75 & 2.0 & 2.75 & 4.05 & 0.95 & 1.3 & 1.45 \\
\hline 73 & 2.25 & 3.2 & 5.0 & 1.05 & 1.4 & 1.6 \\
\hline 71 & 2.5 & 3.65 & 6.05 & 1.15 & 1.55 & 1.75 \\
\hline 69 & 2.8 & 4.3 & 7.6 & 1.2 & 1.65 & 1.9 \\
\hline 67 & 3.3 & 5.75 & 9.85 & 1.25 & 1.75 & 2.05 \\
\hline 65 & 4.6 & 8.0 & 11.55 & 1.3 & 1.85 & 2.15 \\
\hline 63 & 7.35 & 11.0 & 13.5 & 1.35 & 2.0 & 2.4 \\
\hline 61 & 10.1 & 13.0 & 14.25 & 1.55 & 2.35 & 2.75 \\
\hline 59 & 11.85 & 14.35 & 14.7 & 2.05 & 3.15 & 3.7 \\
\hline 57 & 12.95 & 14.95 & 14.95 & 2.75 & 4.05 & 4.75 \\
\hline 55 & 14.45 & 14.95 & 14.95 & 3.35 & 4.8 & 5.5 \\
\hline
\end{tabular}


3.3 - SCHUN characterization

\subsubsection{Method and scenario}

The method used to obtain the GNSS pseudo range error characterization can be split in 3 steps. First the generation of the propagation channel with SCHUN; second the generation of the pseudo ranges and finally the characterization as presented in 3.1.

In order to generate the propagation channel with SCHUN, first the trajectory is defined; second, the satellites location in azimuth and elevation are provided; finally, the virtual city is characterized by the building width, height, etc. All these parameters can be found in [17].

To realistically characterize the pseudo range errors due to SCHUN, it is of the utmost importance to verify that:

- All satellite locations in azimuth and elevation are represented. By doing so, it is certain that the characterization will not be specific to one particular azimuth or elevation value.

- The virtual city and trajectory generated is identical for all generated satellite signal propagation channels

- The satellite signal propagation channel is characterize by its elevation and azimuth, consequently the relative azimuth between the receiver and the transmitter must be constant all over the simulation

Due to these considerations, the trajectory generated is illustrated in Figure 16; by defining a linear trajectory, it ensures a constant relative azimuth all along the trajectory.

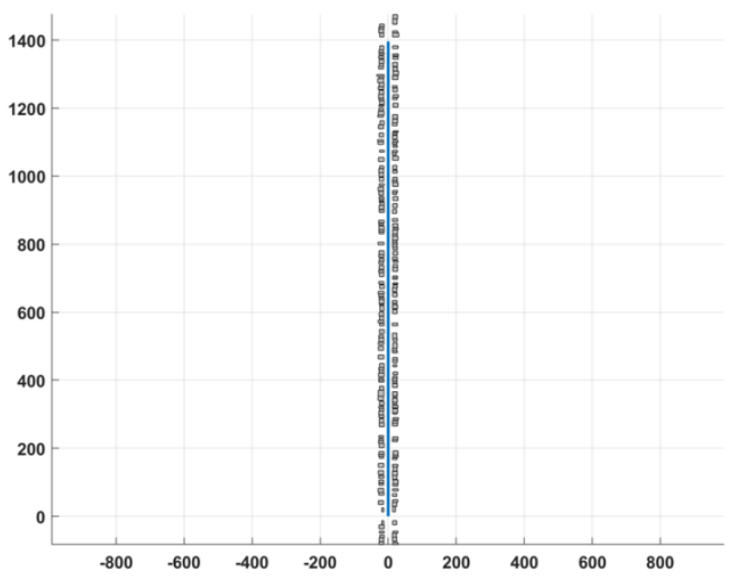

Figure 16 - trajectory considered - ENU coordinates

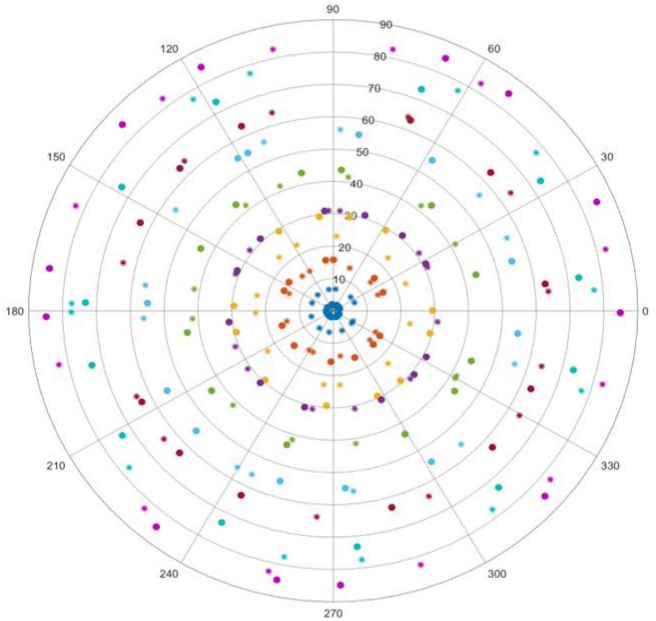

Figure 17 - Satellites generated from a monte carlo to the following one

Regarding the generation of the satellites location, the azimuth and elevation are generated following random distribution over $[0: 360]^{\circ}$ and $[0: 90]^{\circ}$ respectively. In Figure 17, an example of the satellites generated between 2 consecutives Monte Carlo is provided. This process permits to generate all elevation and azimuth combinations over the Monte Carlo simulations.

\subsubsection{Results}

The characterization is made with respect to the $C / N_{0}$ estimated. Results for the fitting characterization are provided in the right part of Figure 18. Results for the over bounding characterization are provided in the left part of Figure 18. As for the QuaDRiGa, there is not much difference between the 3 Gaussian distributions, $N(0, \sigma), N($ mean, $\sigma)$ and $N(\max , \sigma)$. A slight increase of the variances can be observed as the $\mathrm{C} / \mathrm{N} 0$ decreases. 

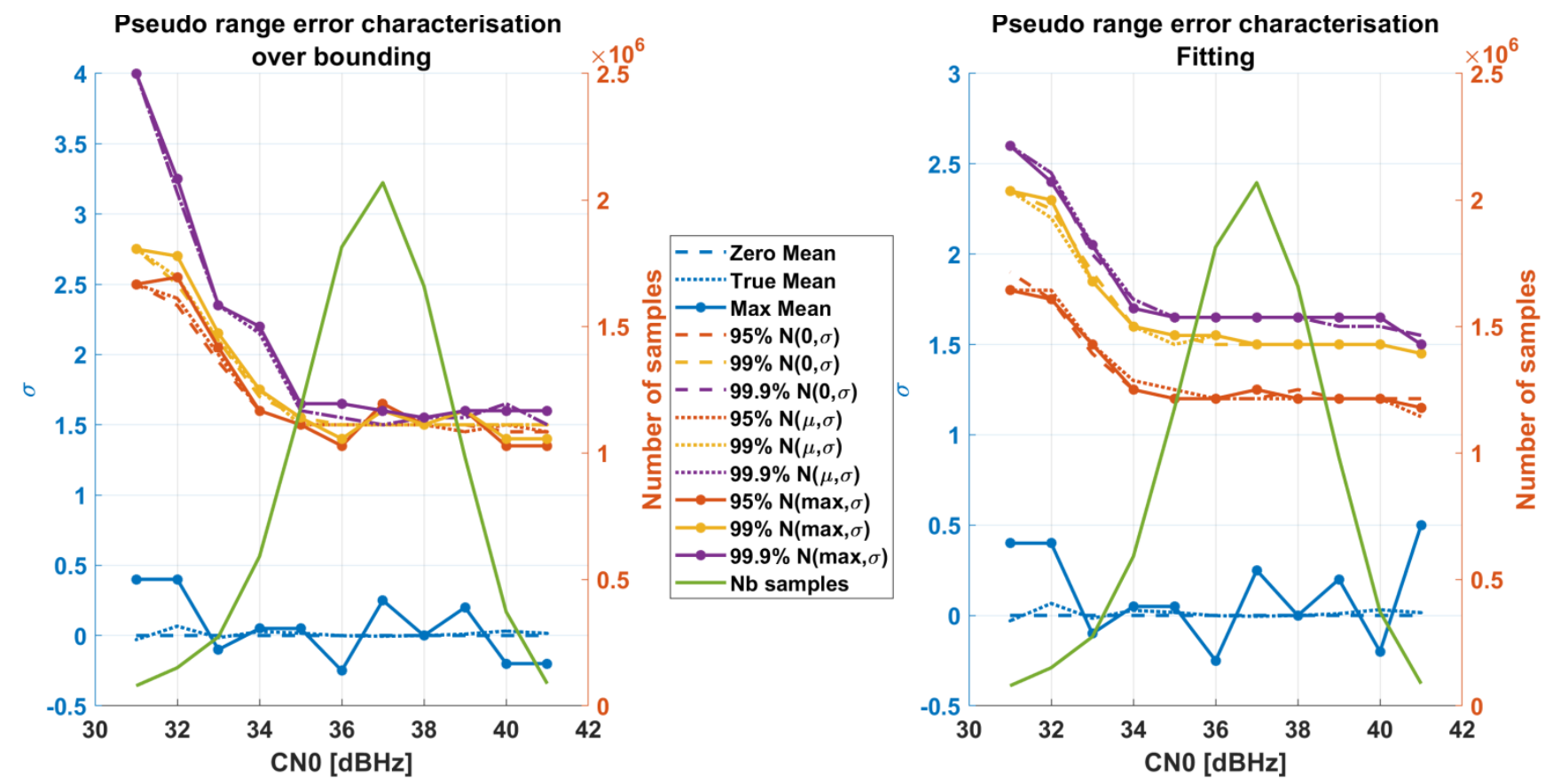

Figure 18 - GNSS characterization

In order to compare the behavior of the over bounding results and the fitting results, Figure 19 is provided. For C/N0 lower than $35 \mathrm{dBHz}$, the over bounding variances are always larger than the fitting variances. The difference increases as the C/N0 decreases.

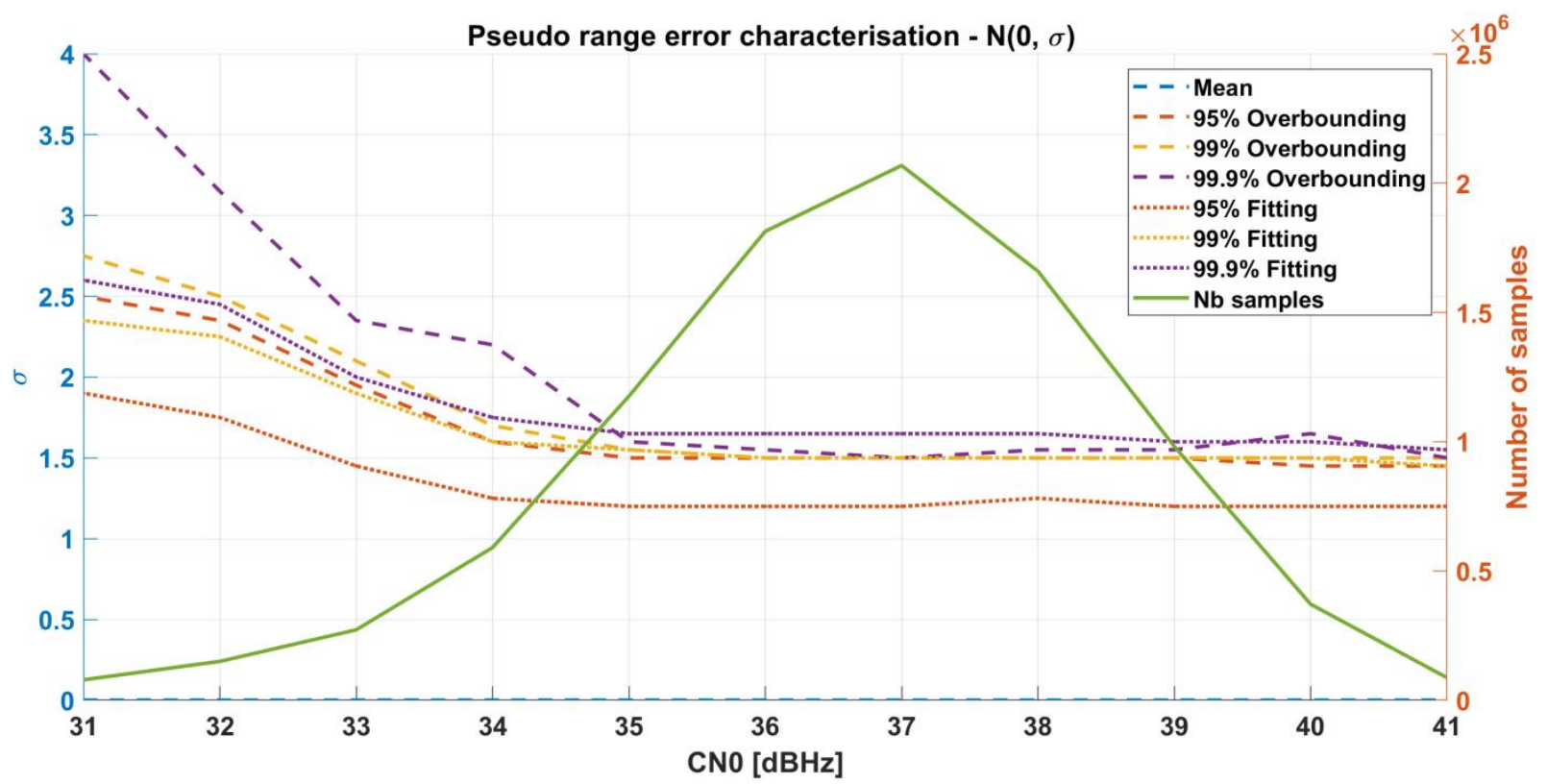

Figure 19 - GNSS characterization

In Table 2, the $\sigma$-values obtained for the $N(0, \sigma)$ are provided. They are used in the simulations of the next sections. More specifically, in the simulation the $\sigma$-values used corresponds to the $99.9 \%$-columns. 
Table 2 - Characterization values obtained for a $N(\mathbf{0}, \sigma)$

\begin{tabular}{|c|c|c|c|c|c|c|}
\hline & \multicolumn{5}{|c|}{$\sigma$} \\
\hline & \multicolumn{3}{|c|}{ Overbounding } & \multicolumn{3}{c|}{ Fitting } \\
\hline$C / N_{0}[\mathrm{dBHz}]$ & $95 \%$ & $99 \%$ & $99.9 \%$ & $95 \%$ & $99 \%$ & $99.9 \%$ \\
\hline 41 & 1.45 & 1.5 & 1.5 & 1.2 & 1.45 & 1.55 \\
\hline 40 & 1.45 & 1.5 & 1.65 & 1.2 & 1.5 & 1.6 \\
\hline 39 & 1.5 & 1.5 & 1.55 & 1.2 & 1.5 & 1.6 \\
\hline 38 & 1.5 & 1.5 & 1.55 & 1.25 & 1.5 & 1.65 \\
\hline 37 & 1.5 & 1.5 & 1.5 & 1.2 & 1.5 & 1.65 \\
\hline 36 & 1.5 & 1.5 & 1.55 & 1.2 & 1.5 & 1.65 \\
\hline 35 & 1.5 & 1.55 & 1.6 & 1.2 & 1.55 & 1.65 \\
\hline 34 & 1.6 & 1.7 & 2.2 & 1.25 & 1.6 & 1.75 \\
\hline 33 & 1.95 & 2.1 & 2.35 & 1.45 & 1.9 & 2.0 \\
\hline 32 & 2.35 & 2.5 & 3.15 & 1.75 & 2.25 & 2.45 \\
\hline 31 & 2.5 & 2.75 & 4.0 & 1.9 & 2.35 & 2.6 \\
\hline
\end{tabular}

\section{IMPLEMENTATION OF HYBRID GNSS/5G NAVIGATION FILTERS}

In this paper, two navigation modules are studied, an Extended Kalman Filter (EKF) and an Unscented Kalman Filters (UKF). Both assume that the errors follow Gaussian distributions which have been accurately characterized in section 3 .

In this section, first the pseudo ranges measurement models for the systems considered in the study are provided. Second the Kalman filter theories are reminded.

\section{1 - Pseudo ranges measurements and time frame considerations}

In this study, measurements from three systems are considered, GPS, Galileo and 5G systems, where each system has its own reference time frame. Therefore, in order to develop the hybrid navigation modules, it is of the utmost importance to express the measurements of each system in the same reference time frame. The GPS time reference frame has been chosen to be the reference time frame for this study.

Two kinds of measurements are considered in this study: pseudo range code measurements for both 5G and GNSS systems and pseudo range rate measurements for GNSS systems. The pseudo range code measurement models are presented in this section. The pseudo range rate models for GNSS systems are well known and mastered.

\subsubsection{GPS Pseudo ranges measurements}

The pseudo range is defined as the difference between the reception time and the transmission time. The pseudo range measured for the $j^{\text {th }}$ GPS satellite by the user is modeled as (13).

Where:

$$
\rho_{G P S_{j}}=c \cdot\left(t_{u s e r, R X}-t_{u s e r, T X}\right)
$$

- $\quad \rho_{G P S_{j}}$ is the pseudo-distance measured for the $j^{\text {th }}$ GPS satellite by the receiver

- $\quad c$ is the speed of the light

- $t_{\text {user }, R X}$ is the reception time expressed in the receiver clock time frame

- $\quad t_{u s e r, T X}=t_{s a t_{j, G P S, T X}}$ is the GPS satellite $j$ emission time assumed by the receiver expressed in the receiver clock time frame

The receiver reference time frame and the GPS satellite time reference frame are not necessarily synchronized. Each time can also be expressed in the selected reference clock time frame: the GPS time frame.

The reception time is expressed in the reference time frame as (14) where $\Delta t_{G P S / \text { user }}$ is the time shift of the receiver clock with respect to the GPS clock time frame, see Figure 20 left part.

$$
t_{\text {user }, R X}=t_{G P S, R X}+\Delta t_{G P S / \text { user }}
$$

The emission time is expressed in the reference time frame as (15) where $\Delta t_{G P S / s a t_{j, G P S}}$ is the time shift of the $j^{\text {th }}$ GPS satellite clock time frame with respect to the GPS clock time frame.

$$
t_{u s e r, T X}=t_{G P S, T X}+\Delta t_{G P S / s a t_{j, G P S}}
$$


The pseudo distance measured for the $j^{\text {th }}$ GPS satellite by the user in GPS time frame is thus modeled as (16) where $r_{G P S_{j}}$ is the geometric distance between the $j^{\text {th }}$ GPS satellite and the receiver.

\subsubsection{Galileo pseudo-distance model}

$$
\rho_{G P S_{j}}=r_{G P S_{j}}+c \cdot \Delta t_{G P S / \text { user }}-c \cdot \Delta t_{G P S / s a t_{j, G P S}}
$$

The pseudo distance measured for the $j^{\text {th }}$ Galileo satellite by the user is modeled as (17).

$$
\rho_{\text {Gal }}=c \cdot\left(t_{u s e r, R X}-t_{u s e r, T X}\right)
$$

Where:

- $\quad \rho_{G a l_{j}}$ is the pseudo-distance measured for the $j^{\text {th }}$ Galileo satellite by the receiver

- $\quad c$ is the speed of the light

- $t_{u s e r, R X}$ is the reception time expressed in the receiver clock time frame

- $\quad t_{u s e r, T X}=t_{s a t}{ }_{j, G a l, T X}$ is the emission time assumed by the receiver expressed in the receiver clock time frame

The receiver clock time frame and the satellite clock time frame are not necessarily synchronized. Each time must be expressed in the reference clock time frame: the GPS clock time frame.

The reception time is expressed in the reference time frame as (18) where $\Delta t_{G P S / u s e r}$ is the time shift of the receiver clock with respect to the GPS clock time frame, see Figure 20 middle part.

$$
t_{u s e r, R X}=t_{G P S, R X}+\Delta t_{G P S / u s e r}
$$

The emission time is expressed in the reference time frame as (19), where $\Delta t_{G P S / s a t_{j, g a l}}$ is the time shift of the $j^{t h}$ satellite clock with respect to the GPS clock time frame.

$$
t_{u s e r, T X}=t_{G P S, T X}+\Delta t_{G P S / s_{a t}, g a l}
$$

The pseudo distance measured for the $j^{\text {th }}$ Galileo satellite by the user in GPS time frame is thus modeled as (20) where $r_{G a l_{j}}$ is the geometric distance between the $j^{\text {th }}$ Galileo satellite and the receiver.

$$
\rho_{G a l_{j}}=r_{G a l_{j}}+c \cdot \Delta t_{G P S / \text { user }}-c \cdot \Delta t_{G P S / s a t_{j, G a l}}
$$

The time shift $\Delta t_{G P S / s a t_{j, G a l}}$ can be expressed as: $\Delta t_{G P S / s a t_{j, G a l}}=\Delta t_{G P S / G a l}+\Delta t_{G a l / s a t_{j, G a l}}$; where $\Delta t_{G P S / G a l}$ represents the time shift between the GPS and Galileo systems and $\Delta t_{G a l / s a t_{j, G a l}}$ is the time shift of the $j^{\text {th }}$ Galileo satellite clock time frame with respect to the Galileo clock time frame.

\subsection{3 $5 \mathrm{G}$ pseudo-distance model}

$$
\rho_{\text {Gal }_{j}}=r_{G a l_{j}}+c \cdot \Delta t_{G P S / \text { user }}-c \cdot \Delta t_{G P S / G a l}-c \cdot \Delta t_{G a l / s a t_{j, G a l}}
$$

The $5 \mathrm{G}$ pseudo distance measured for the $j^{\text {th }}$ Base Station by the user is modeled as (22).

$$
\rho_{5 G_{j}}=c \cdot\left(t_{u s e r, R X}-t_{u s e r, T X}\right)
$$

Where:

- $\quad \rho_{5 G_{j}}$ is the pseudo-distance measured for the $j^{\text {th }}$ Base Station by the receiver

- $\quad c$ is the speed of the light

- $\quad t_{u s e r, R X}$ is the reception time expressed in the receiver clock time frame

- $\quad t_{u s e r, T X}=t_{B S_{j}, T X}$ is the emission time assumed by the receiver expressed in the receiver clock time frame

The receiver clock time frame and the Base Station time frame are not necessarily synchronized. Each time must be expressed in the reference clock time frame: the GPS clock time frame.

The reception time is expressed in the reference time frame as (23) where $\Delta t_{G P S / u s e r}$ is the time shift of the receiver clock with respect to the GPS clock time frame, see Figure 20 right part.

$$
t_{\text {user }, R X}=t_{G P S, R X}+\Delta t_{G P S / \text { user }}
$$

The emission time is expressed in the reference time frame as (24) $\mathrm{w}$ here $\Delta t_{G P S / B S_{j}}$ is the time shift of the $j^{t h}$ Base station clock with respect to the GPS clock time frame.

$$
t_{u s e r, T X}=t_{G P S, T X}+\Delta t_{G P S / B S_{j}}
$$

The pseudo distance measured for the $j^{\text {th }}$ Base Station by the user in GPS time frame is thus modeled as (25) where $r_{5 G_{j}}$ is the geometric distance between the $j^{\text {th }} 5 \mathrm{G}$ base station and the receiver.

$$
\rho_{5 G_{j}}=r_{5 G_{j}}+c \cdot \Delta t_{G P S / \text { user }}-c \cdot \Delta t_{G P S / B S_{j}}
$$


The time shift $\Delta t_{G P S / B S_{j}}$ can be expressed as: $\Delta t_{G P S / B S_{j}}=\Delta t_{G P S / 5 G}+\Delta t_{5 G / B S_{j}}$; where $\Delta t_{G P S / 5 G}$ represents the time shift between the GPS and $5 \mathrm{G}$ systems and $\Delta t_{5 G / B S_{j}}$ is the time shift of the $j^{\text {th }} 5 \mathrm{G}$ Base Station clock time frame with respect to the $5 \mathrm{G}$ clock time frame. See Figure 20 right part (a) and (b).

\subsubsection{Pseudo ranges measurements models summary}

$$
\rho_{5 G_{j}}=r_{G a l_{j}}+c \cdot \Delta t_{G P S / \text { user }}-c \cdot \Delta t_{G P S / 5 G}-c \cdot \Delta t_{5 G / B S_{j}}
$$

The set of pseudo ranges considered can be expressed as:

$$
\left\{\begin{array}{cc}
\rho_{G P S_{j}}=r_{G P S_{j}}+c \cdot \Delta t_{G P S / \text { user }}-c \cdot \Delta t_{G P S / s a t_{j, G P S}} & j=1 \ldots N_{G P S} \\
\rho_{G a l_{j}}=r_{G a l_{j}}+c \cdot \Delta t_{G P S / \text { user }}-c \cdot \Delta t_{G P S / G a l}-c \cdot \Delta t_{G a l / \text { sat }_{j, G a l}} & j=1 \ldots N_{G a l} \\
\rho_{5 G_{j}}=r_{G a l_{j}}+c \cdot \Delta t_{G P S / \text { user }}-c \cdot \Delta t_{G P S / 5 G}-c \cdot \Delta t_{5 G / B S_{j}} & j=1 \ldots N_{5 G}
\end{array}\right.
$$

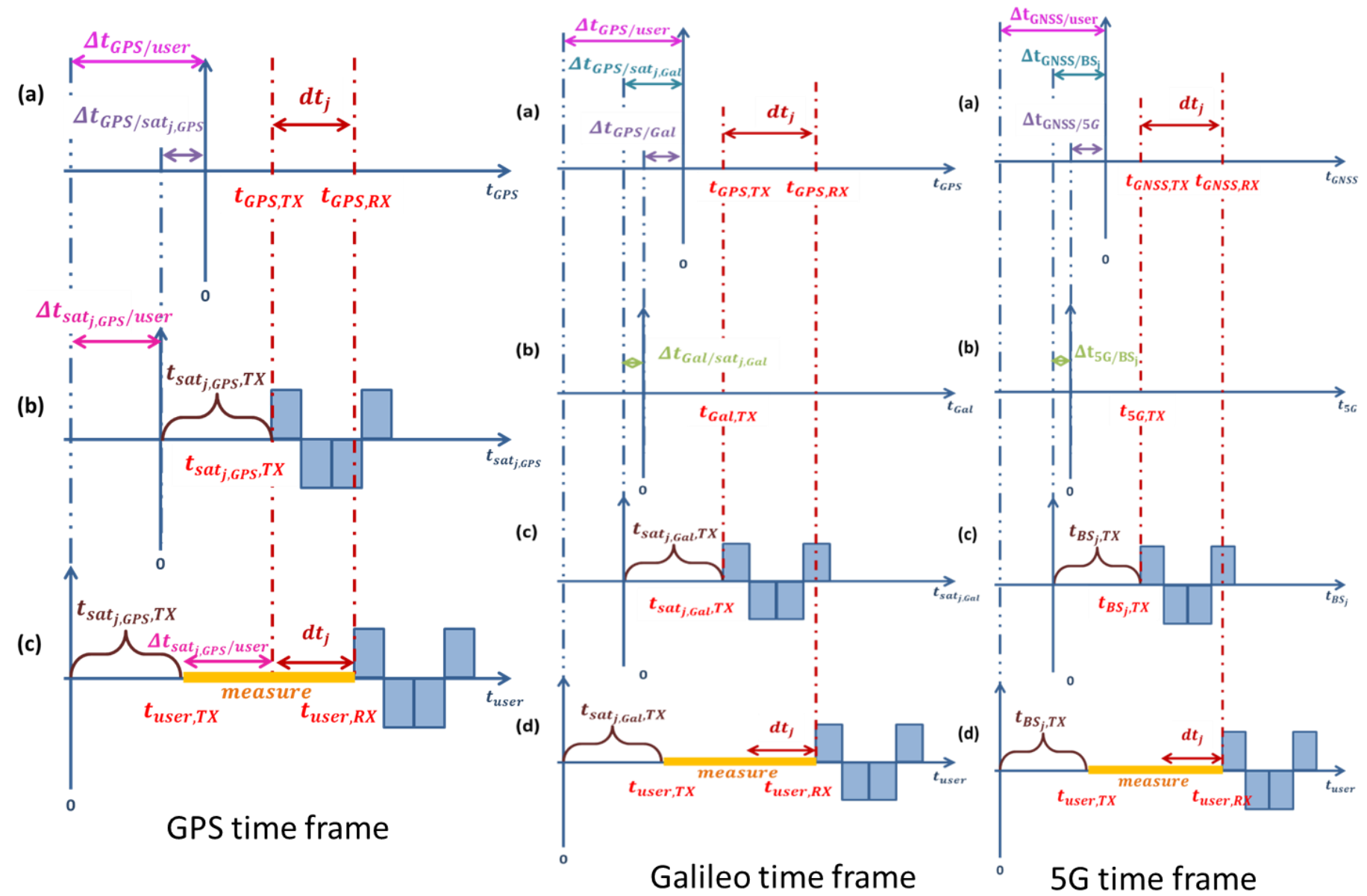

Figure 20 - Measurements time frame consideration

Several hypotheses will be considered and tested regarding the biases and synchronization errors (equal to zero, constant, evolving). In particular, the following filters are derived assuming that GNSS measurements are corrected, thus the offsets $\Delta t_{G P S / s a t_{j, G P S}}$ and $\Delta t_{G a l / s a t_{j, G a l}}$ are perfectly corrected and thus equal 0 . We also assume that the $5 \mathrm{G}$ time offset $\Delta t_{5 G / B S_{j}}$ can be considered constant over the observation duration and for simplification purposes it is set to 0 .

\section{2 - Hybrid navigation filters presentation}

The objective of the study is to characterize the performances of EKF and UKF hybrid navigation filters using 5G and GNSS measurements in a realistic multipath environment. In order to quantify those performances, the hybrid navigation filters will be compared to the standalone navigation solutions. In this section the hybrid navigation filters theory are presented; note that the standalone navigation filters are not presented, however, the only difference with the hybrid navigation filters lies in the measurements considered. 


\subsubsection{System model presentation}

First the system model is detailed, second the EKF theory is presented and third the UKF is presented.

Classically, the system is modelled with the following non-linear equations:

Where:

$$
\left\{\begin{array}{c}
X_{k+1}=f\left(X_{k}\right)+W_{k} \\
Y_{k}=h\left(X_{k}\right)+V_{k}
\end{array}\right.
$$

- $f$ and $h$ are the nonlinear dynamic and observation functions

- $X_{k}$ is the state vector

○ $Y_{k}$ is the measurement vector

$\circ V_{k}$ is the measurement noise vector

$\circ h\left(X_{k}\right)$ is observation matrix $h\left(X_{k}\right)=\left[\begin{array}{c}h^{(G P S)}\left(X_{k}\right) \\ h^{(G a l)}\left(X_{k}\right) \\ h^{(5 G)}\left(X_{k}\right) \\ \dot{h}^{(G P S)}\left(X_{k}\right) \\ \dot{h}^{(G a l)}\left(X_{k}\right)\end{array}\right]$

$\circ \quad h^{(X)}$ are pseudo range code measurements defined in (27)

- $\dot{h}^{(X)}$ are pseudo range rate measurements

$\circ \quad$ The command vector is assumed null.

According to (27), the state vector to be estimated is thus

$$
X_{k}=\left[\begin{array}{llllllllll}
X & V_{X} & Y & V_{Y} & Z & V_{Z} & c \cdot \Delta t_{G P S / \text { user }} & c \cdot \Delta t_{G P S / \text { user }} & c \cdot \Delta t_{G P S / G a l} & c \cdot \Delta t_{G P S / 5 G}
\end{array}\right]
$$

Where $\left[\begin{array}{lll}X & Y & Z\end{array}\right]$ and $\left[\begin{array}{lll}V_{X} & V_{Y} & V_{Z}\end{array}\right]$ represent respectively the position and velocity of the user.

\subsubsection{The extended Kalman Filter}

The EKF is a classical navigation filter; it is based on the analytical method for the evaluation of the integrals over the Gaussian weighted non-linear functions consists in using Taylor polynomial expansion about a single point. The EKF gives reasonable estimation results if the nonlinearities are not very severe. Moreover, the Kalman Filter is a Bayesian estimation technique where all probability density functions are supposed Gaussian. Theoretical derivation can be found in [24], the main steps are reminded here.

The EKF provides at each instant the MMSE estimator, which minimizes the mean square at every epoch, $k$. At each epoch the EKF proceeds in 2 stages, see Figure 21

$\circ$ A state prediction

$\circ$ A measurement update

At epoch $k+1$, there is an intermediate state estimate just after the prediction step, named the a priori state, which will be indexed by $k+1 \mid k$. After a measurement update, the new estimate is called a posteriori state and will be indexed by $k+1 \mid k+1$. Those steps are summarized in Figure 21.

Being an MMSE, the EKF output is defined as (30) and the a priori state covariance matrix is defined as (31).

$$
\begin{gathered}
\hat{X}_{k \mid k}=E\left[X_{k} \mid Y_{0}, \ldots, Y_{k}\right]=E\left[X_{k} \mid Y_{0}^{k}\right] \\
\Sigma_{k+1 \mid k}=\operatorname{cov}\left[X_{k+1} \mid Y_{0}^{k}\right]
\end{gathered}
$$

This a priori state covariance matrix is also the a priori estimation error covariance matrix. The same goes for the a posteriori state covariance matrix. Both are used to have an indicator of the estimator's quality. 
In order to compute a priori and a posteriori state and covariance, the model (28) must be linearized. Considering $\hat{X}_{k \mid k}$ and $\hat{X}_{k \mid k-1}$ recent and reasonably accurate approximations of $X_{k}$, it is possible to develop the functions $f$ and $h$ in Taylor series at the neighbourhood of $\hat{X}_{k \mid k}$ and $\hat{X}_{k \mid k-1}$ respectively.

$$
\left\{\begin{array} { c } 
{ f ( X _ { k } ) = f ( \hat { X } _ { k | k } ) + \frac { \partial f } { \partial X _ { k } X _ { k } = \hat { X } _ { k | k } } ( X _ { k } - \hat { X } _ { k | k } ) } \\
{ h ( X _ { k } ) = h ( \hat { X } _ { k | k - 1 } ) + \frac { \partial h } { \partial X _ { k | X _ { k } = \hat { X } _ { k | k - 1 } } } ( X _ { k } - \hat { X } _ { k | k - 1 } ) }
\end{array} \text { noting } \left\{\begin{array}{c}
F_{k}=\frac{\partial f}{\partial X_{k} X_{k}=\hat{X}_{k \mid k}} \\
H_{k}=\frac{\partial h}{\partial X_{k}}{ }_{X_{k}=\hat{X}_{k \mid k-1}}
\end{array}\right.\right.
$$

Where $F_{k}$ and $H_{k}$ are the Jacobian matrix of $f$ and $h$. The model used for $F_{k}$ is well known and can be found in [24].

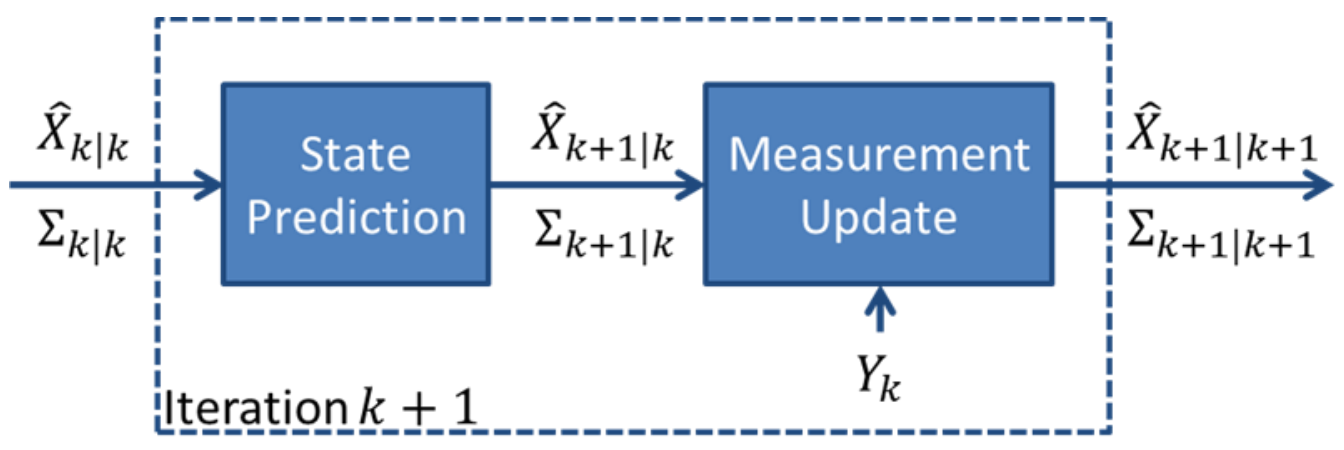

Figure 21 - EKF processing steps

Using the linearized model, the equation defining the EKF are provided in equation (33) to (37). The prediction step is defined by (33) and (34).

$$
\begin{gathered}
\hat{X}_{k+1 \mid k}=f\left(\hat{X}_{k \mid k}\right) \quad \text { state prediction } \\
\Sigma_{k+1 \mid k}=F_{k} \Sigma_{k \mid k} F_{k}{ }^{T}+Q_{k} \quad \text { a priori covariance }
\end{gathered}
$$

An important feature must be defined: the Kalman gain $K_{k+1}$, see (35). This gain permits to balance the information coming from the state prediction and the observations. If the state prediction is deemed good; the $K_{k+1}$ will be small (the model is trusted). If the state prediction is deemed bad, the $K_{k+1}$ will be large (more confidence are given to the measurements).

$$
K_{k+1}=\Sigma_{k+1 \mid k} H_{k+1}^{T}\left(H_{k+1} \Sigma_{k+1 \mid k} H_{k+1}^{T}+R_{k+1}\right)^{-1} \quad \text { Kalman gain }
$$

The a posteriori equation are provided in (36) and (37).

$$
\begin{gathered}
\hat{X}_{k+1 \mid k+1}=\widehat{X}_{k+1 \mid k}+K_{k+1}\left(Y_{k+1}-h\left(\widehat{X}_{k+1 \mid k}\right)\right) \text { state update } \\
\Sigma_{k+1 \mid k+1}=\Sigma_{k+1 \mid k}-K_{k+1} H_{k+1} \Sigma_{k+1 \mid k} \quad \text { a posteriori covariance }
\end{gathered}
$$

\subsubsection{The Unscented Kalman Filters}

The UKF is based on a statistical linearization technique; technique to tackle Gaussian weighted non-linear function instead of the analytical one used in EKF. This technique consists in the linearization of a nonlinear function of a random variable through a linear regression between $n$ points drawn from the prior distribution of the random variable. In other words, the UKF is based on a deterministic sampling approach; the state distribution is represented using a minimal set of carefully chosen sample points called sigma points. It must also be noted that for highly non-linear model, the UKF outperforms the EKF

The UKF is founded on the intuition that it is easier to approximate a probability distribution than to approximate an arbitrary nonlinear function or transformation. The sigma points are chosen so that their mean and covariance are exactly $\hat{X}_{k \mid k}$ and $\Sigma_{k \mid k}$. Each sigma point is then propagated through the nonlinearity yielding in the end a cloud of transformed points. The new estimated mean and covariance are then computed based on their statistics. This process is called unscented transformation. point $X_{k}^{i}$.

The set of sigma points is defined as (38) where $n$ is the dimension of the state vector and $w_{i}$ is the weight associated to

$$
X_{k}=\left\{\left(w_{i}, X_{k}^{i}\right), i=0, \ldots, 2 n\right\}
$$

There are several ways to select sigma point, in the following the unscented transformation is provided; the other main selection scheme is called the scaled unscented transformation. With the unscented transformation, the sigma points are defined such that: 


$$
\left\{\begin{array}{c}
X_{k}^{0}=\hat{X}_{k \mid k} \quad-1<w_{0}<1 \\
X_{k}^{i}=\hat{X}_{k \mid k}+\left(\sqrt{\frac{n}{1-w_{0}} \Sigma_{k \mid k}}\right)_{i} w_{i}=\frac{1-w_{0}}{2 n} \quad \forall i=1, \ldots, n \\
X_{k}^{i}=\hat{X}_{k \mid k}-\left(\sqrt{\frac{n}{1-w_{0}} \Sigma_{k \mid k}}\right)_{i} w_{i}=\frac{1-w_{0}}{2 n} \quad \forall i=n+1, \ldots, 2 n
\end{array}\right.
$$

Where $\left(\sqrt{\frac{n}{1-w_{0}} \Sigma_{k \mid k}}\right)$ is the $i^{\text {th }}$ row or column of the matrix $\sqrt{\frac{n}{1-w_{0}} \Sigma_{k \mid k}}$.

The weights are such that: $\sum_{i=0}^{2 n} w_{i}=1$

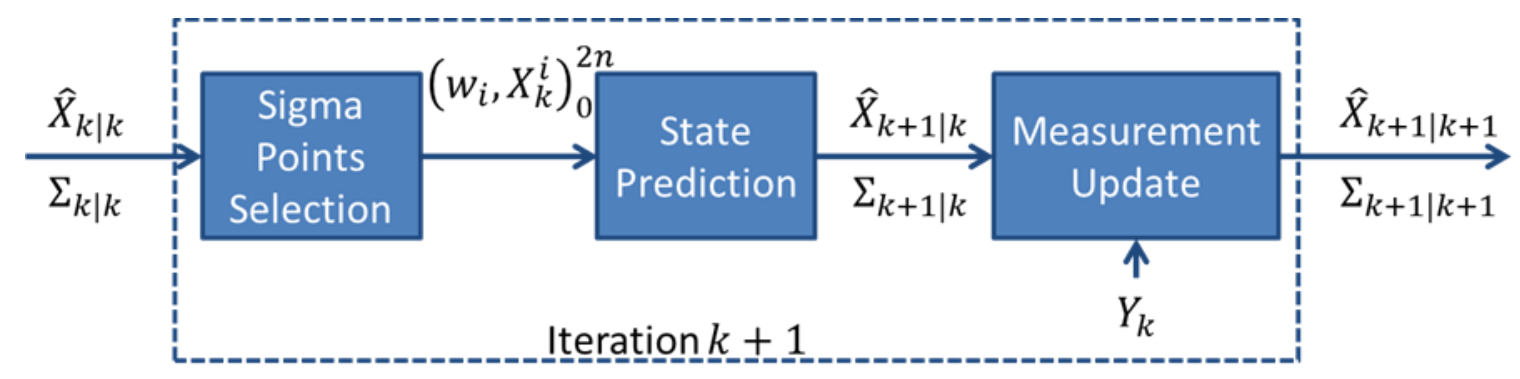

Figure 22 - UKF processing steps

The set of equation summarizing the UKF processing are provided in equations (40) to (42). The state update and the a priori covariance update equation for the UKF are identical to the EKF and are provided in (36) and (37).

$$
\begin{gathered}
\hat{X}_{k+1 \mid k} \cong \sum_{j=0}^{2 n} w_{j} f\left(X_{k}^{j}, W_{k}\right) \text { state prediction } \\
\Sigma_{k+1 \mid k} \cong \sum_{j=0}^{2 n} w_{j}\left[f\left(X_{k}^{j}\right)-\hat{X}_{k+1 \mid k}\right]\left[f\left(X_{k}^{j}\right)-\hat{X}_{k+1 \mid k}\right]^{T}+Q_{k} \quad \text { a priori covariance } \\
K_{k+1}=\Sigma_{k+1 \mid k}^{X Y}\left(\sum_{k+1 \mid k}^{Y Y}\right)^{-1} \quad \text { Kalman gain }
\end{gathered}
$$

Finally, it must be noted that there is a tricky part when implementing the UKF (both standalone and hybrid). According to (39) the definition of the sigma points in the UKF requires the determination of the square root matrix of the covariance matrix. In the literature, [25], several methods are proposed to compute this square root matrix: the Cholesky decomposition method, the Diagonalization method, the SCHUR method, etc. During the study, both the Cholesky and the diagonalization methods have been tested. Both, from time to time, leads to divergence issue due to numerical issues; thus, basically, the positioning solution availability is higher with the EKF than with the UKF.

In this section, the navigation modules used in the study have been presented; details can be found in [24].

\section{OVERVIEW OF THE SIMULATOR}

As already explained, in order to generate the pseudo ranges and to characterize the positioning performances of GNSS and 5G signals a simulator of 5G and GNSS signals is designed. This section aims at presenting the simulator used to test and validate all models presented until now. In order to do so, first the interest of a simulator is briefly reminded. Second, the process of the simulator is detailed.

\section{1 - Interest of a simulator}

As noted before, GNSS systems are mature and well known systems; however, 5G technology is being currently standardized and its positioning performance, as well as a potential generic receiver scheme to conduct positioning operations, is still under analysis. Therefore, to study the potential capabilities provided by $5 \mathrm{G}$ systems and to develop and to compare 5G, GNSS and hybrid 5G/GNSS signals generic positioning module schemes, one possible way forward consists in developing a controlled 5G and GNSS signals environment for positioning; or in other words, a 5G/GNSS signals positioning simulator. The main advantages of using a simulator instead of real signals are twofold. The first main advantage is the possibility to control the environment and thus to control the exact impairments the 5G and GNSS signals positioning module will have to overcome. The 
second main advantage is the possibility to control and to completely monitor the behavior of the implemented signal processing and positioning algorithms. However, the use of a simulator also entails some constraints: this controlled environment must be realistic enough in terms of 5G and GNSS simulated signals, which must be in accordance with the standards, and realistic enough in terms of propagation channel model; the simulated propagation channel must fulfil the $5 \mathrm{G}$ standard requirements defined in the 3GPP standards and the GNSS standards. One additional advantage of a simulator with respect to the process of real signals is the simplification or, directly, the removal of some signal processing stages if their output mathematical model is well characterized: instead of simulating the behavior of the signal processing stage, the output can be directly generated from its mathematical model.

\section{2 - Simulator}

The implemented simulator can be split into 3 modules as illustrated in Figure 23

- $\quad$ A GNSS module named GeneIQ in Figure 23

- A 5G module

- A navigation module

The simulator is provided with the propagation channels generated with SCHUN for GNSS and QuaDRiGa.

The implemented simulator generates 5G and GNSS correlator outputs in multipath environments from their mathematical models, reminded in section 2 and derived in [10] for 5G systems and in [11] for GNSS systems. In order to simplify the simulator and to remove computationally-heavy signal processing stages, only the correlator outputs are considered. Previous signal processing stages are not generated since their impact can be directly generated from the known correlator output mathematical model.

\subsubsection{GeneIQ}

In order to generate GNSS correlator outputs, the simulator first generates the observables or more specifically the delays between the receiver and satellites. To do so, the simulator computes the position of the satellites in view, based on the decoding of the provided Almanach. Then, based on the mask angle and the receiver position, it determines if the satellites are visible from the receiver. Based on models, an ionospheric, a tropospheric and a clock error delays are added to the true distance. Pseudo range code and pseudo range rate measurements are then generated following (43) and (44). Thus the delays and phases of the input signals to be tracked are defined.

$$
\begin{gathered}
P_{k}^{j}=\rho_{\text {GPS }_{j_{k}}}+d_{\text {Iono }_{k}}^{j}+d_{\text {Tropo }_{k}}^{j}+c \cdot\left(\Delta t_{{\text {GPS } / \text { user }_{k}}_{k}}-\Delta t_{{\text {GPS } / \text { sat }_{j, G P S}}_{k}}\right) \\
\varphi_{k}^{j}=\rho_{\text {GPS }_{j_{k}}}-d_{\text {Iono }_{k}}^{j}+d_{\text {Tropo }_{k}}^{j}+c \cdot\left(\Delta t_{{\text {GPS } / \text { user }_{k}}}-\Delta t_{{\text {GPS } / \text { sat }_{j, G P S}}_{k}}\right)
\end{gathered}
$$

Where:

- $\quad \rho_{G P S_{j}}$ is the geometric distance between the satellite and the user for sat $\mathrm{j}$ based on the Ephemerides information from the Almanach: $\rho_{G P S_{j_{k}}}=\sqrt{\left(x_{j_{k}}-x_{u_{\text {ser }}}\right)^{2}+\left(y_{j_{k}}-y_{\text {user }_{k}}\right)^{2}+\left(z_{j_{k}}-z_{u_{\text {ser }}}\right)^{2}}$

- $\quad d_{\text {Iono }_{k}}^{j}$ is the Current true Ionospheric delay [m] for sat $\mathrm{j}$

- $\quad d_{\text {Tropo }_{k}}^{j}$ is the Current true tropospheric delay [m] for sat $\mathrm{j}$

- $\quad \Delta t_{G P S / \text { user }_{k}}$ is the Current user clock bias [s] - TCXO model

- $\Delta t_{G P S / s a t_{j, G P S}}$ is the Current Satellite time clock error [s] - Rubidium model for sat $\mathrm{j}$

Each satellite is then processed independently in order to compute correlator outputs, which models are provided in the previous section. The correlator outputs are then fed to a code and a carrier tracking loops allowing the estimation of delays and phases. The tracking loops delay and phase estimations are then used to generate pseudo range codes and pseudo range rates required to the design of the navigation modules. The process is illustrated in Figure 23.

The pseudo ranges generated are used in the standalone navigation module; an EKF is implemented.

\subsubsection{G module}

In order to compute the correlator outputs, the simulator is provided with data files generated previously with QuaDRiGa. Two files are generated per Base Stations; one containing the delays information for each path, i.e., the delay between the BS and the receiver at each instant and for each path composing the propagation channel. The second file contains the complex amplitudes for each path at each instant.

Then correlator outputs are generated following (9). The correlator outputs are then fed to a code tracking loop allowing the estimation of delays. No carrier tracking loops are implemented for 5G systems; previous theoretical studies have proved that the delay degradation due to a frequency estimation error for $5 \mathrm{G}$ signals can be neglected.

The tracking loops delay estimations are then used to generate pseudo range codes required to the design of the navigation modules. The process is illustrated in Figure 23. The pseudo ranges generated are used in the standalone navigation module; an EKF and an UKF are implemented. 


\subsubsection{Hybrid navigation module}

Based on the GNSS and 5G pseudo ranges generated, 2 hybrid navigation filters are designed: an EKF and an UKF. The process is illustrated in Figure 23.

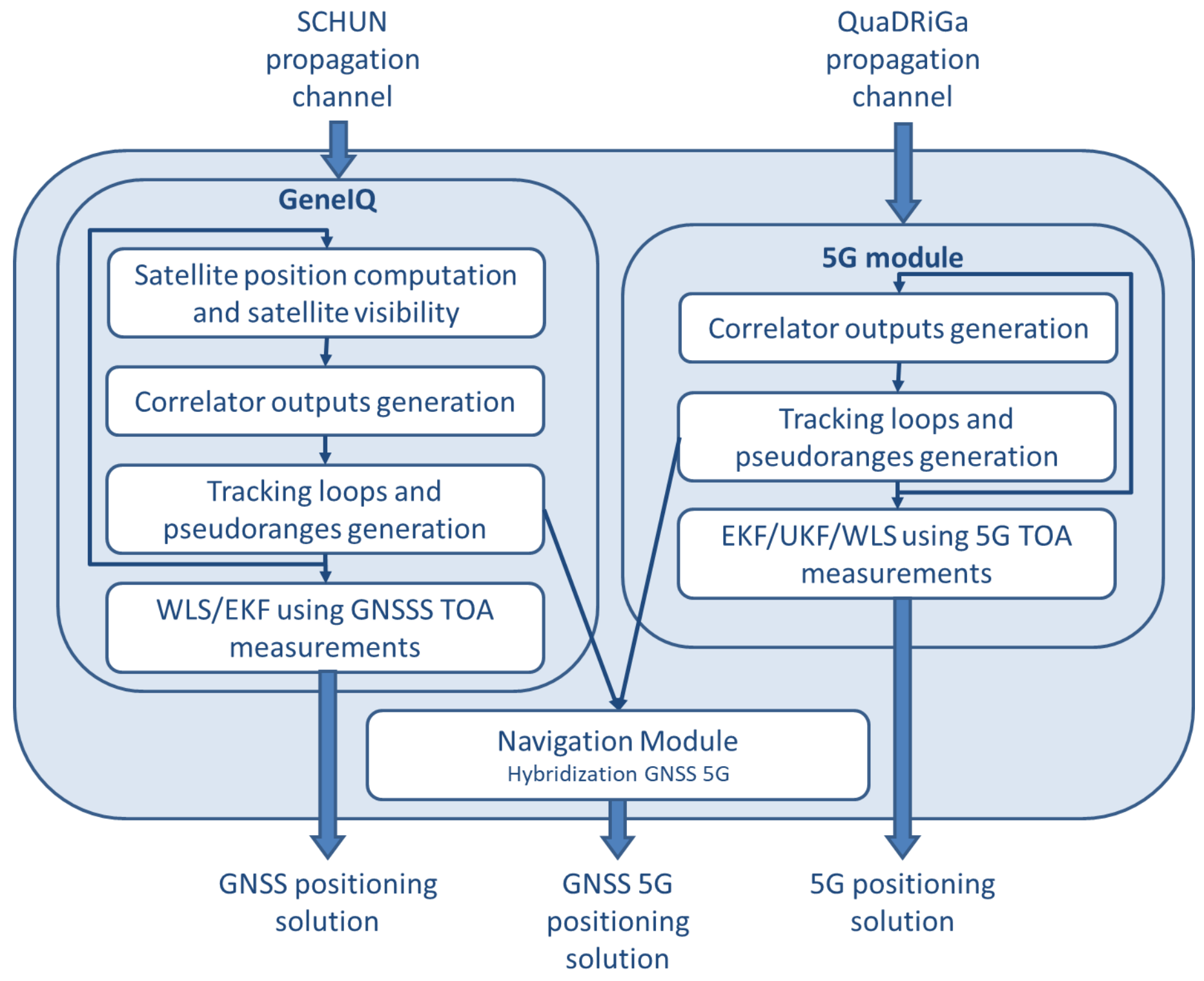

Figure 23 - Simulator overview

\section{RESULTS}

The positioning performances obtained using the previously describe navigation filters are studied and compared for several scenarios,

- $5 \mathrm{G}$ or GNSS standalone navigation solution and hybrid navigation solution.

- Propagation channel: AWGN, LOS and LOS plus multipath

- Fitting and over bounding distribution characterization

By inspecting these different scenarios, the impact of a constrained environment will be analyzed as well as the potential benefit of using SoO. First the scenario considered is presented, second the results are detailed.

\section{1 - Scenario presentation}

First the trajectory is presented, second the GNSS scenario is presented and finally the $5 \mathrm{G}$ scenario is presented. 


\subsubsection{Trajectory}

In order to study the mathematical models developed all along this paper, the scenario used is presented in Figure 24. The trajectory lasts 60 seconds. The receiver is moving at a constant speed of $v=50 \mathrm{~km} / \mathrm{h}$.

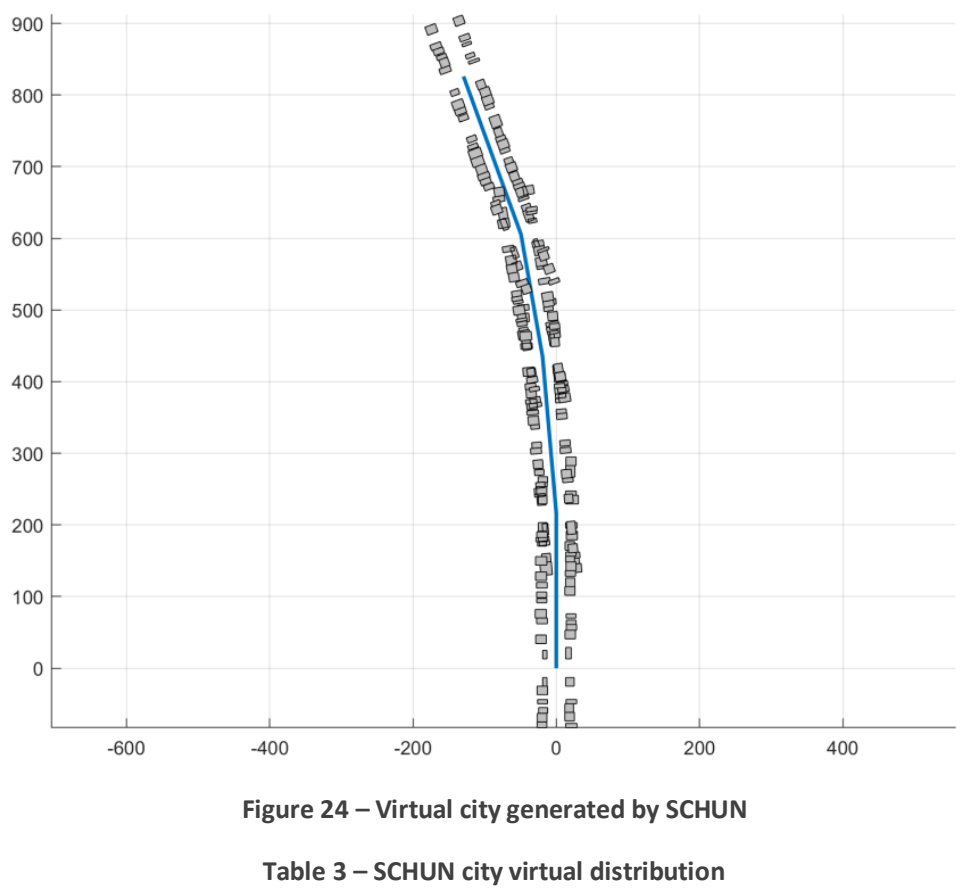

\begin{tabular}{|c|c|c|c|c|}
\hline Parameters & Description & Format & Units & Value \\
\hline Road crossing & Uniform distribution of the street lengths in meters & {$[$ Min Max $]$} & {$[\mathrm{m}]$} & {$[150300]$} \\
\hline Street orientation & $\begin{array}{c}\text { Uniform distribution of the orientation in degrees between two } \\
\text { consecutive streets. }\end{array}$ & {$[$ Min Max $]$} & {$[\mathrm{m}]$} & {$[-2020]$} \\
\hline Building density & The building occupation density in the virtual city & Float & $0<$ index $<1$ & 0.75 \\
\hline Building height & The building height distribution assuming a Gaussian distribution & {$[$ Mean std] } & {$[\mathrm{m}]$} & {$[1520]$} \\
\hline Building width & The building width distribution assuming a Gaussian distribution & {$[$ Mean std] } & {$[\mathrm{m}]$} & {$[1030]$} \\
\hline Building depth & The building depth distribution assuming a Gaussian distribution & [Mean std] & {$[\mathrm{m}]$} & {$[1520]$} \\
\hline Buiding space & Distance between buildings assuming a Gaussian distribution. & [Mean std] & {$[\mathrm{m}]$} & {$[03]$} \\
\hline Building back & The distance between the buildings and the street in meters & {$[$ Min Max $]$} & {$[\mathrm{m}]$} & {$\left[\begin{array}{lll}12 & 14] \\
\hline\end{array}\right.$}
\end{tabular}

\subsubsection{GNSS scenario}

Regarding the GNSS scenario considered, the randomly selected Almanac, for Toulouse city center earth location, gives the sky plot provided in Figure 25. Assuming the virtual city presented in Figure 24 and following the parameters presented in Table 3, a $50^{\circ}$ elevation mask is used, illustrated by the blue disk in Figure 25. Based on this, a maximum of 2 GPS satellites are visible and 3 Galileo satellites. The number of visible satellites can be lower due to the propagation channel generated by SCHUN which can makes the receiver loose the tracking of the satellites. 


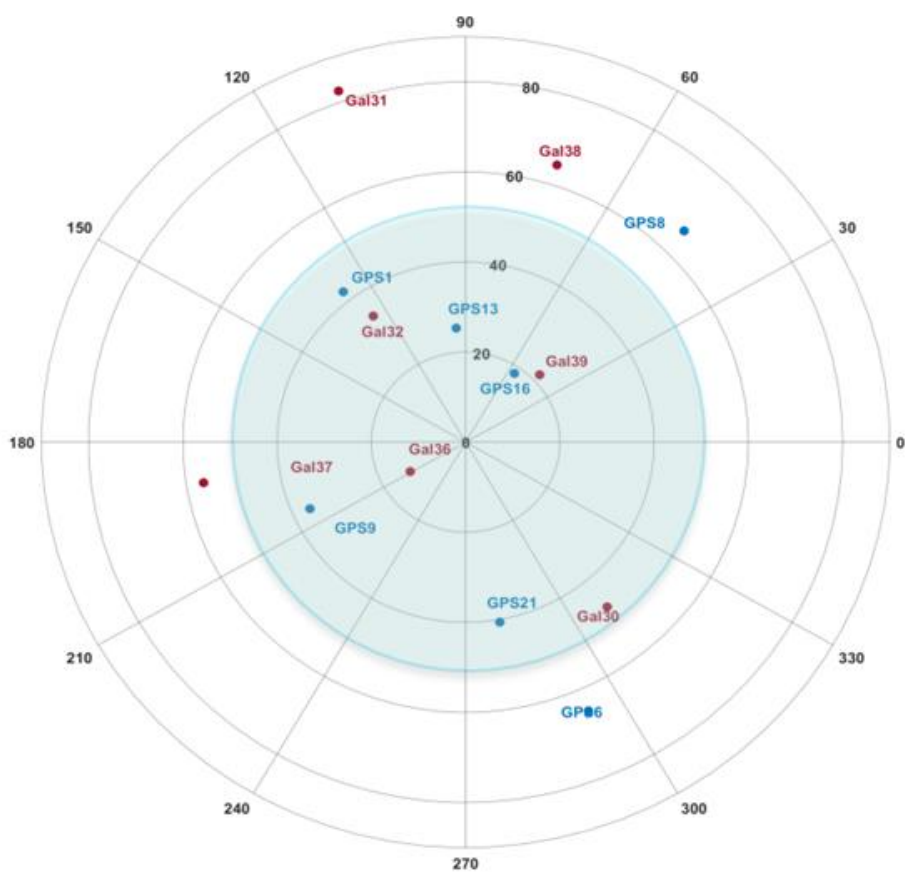

Figure 25 - GNSS sky plot

\subsubsection{G scenario}

Regarding the 5G scenario adopted for the simulations, an Ultra Dense Network is assumed. Basically, the scenario of deployment envisioned consists in BS located at each lamp post of the main and crossing streets. The scheme is provided in Figure 26. Base stations are $15 \mathrm{~m}$ high. The propagation channel is generated assuming the 3GPP_38.901_UMi_LOS high level scenario. The carrier frequency is set to $2 \mathrm{GHz}$.

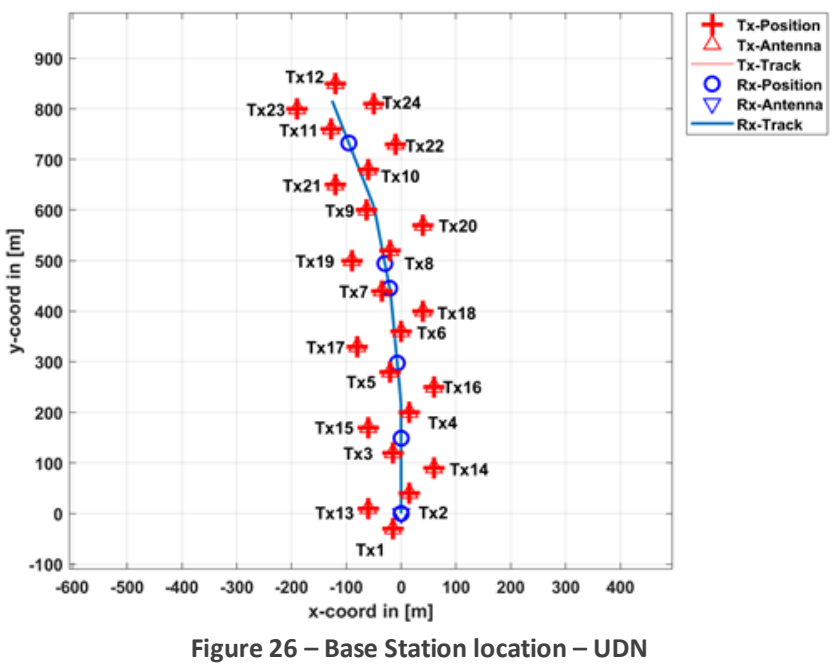

\subsubsection{Configurations tested}

In order to characterize the performances of the navigation filters, four cases are studied for $5 \mathrm{G}$ systems.

- AWGN; In order to have a reference, the first case is based on an AWGN channel; the channel is similar to a pure delay.

- LOS: The channel is assumed composed of one path only, the LOS, the path parameters are evolving in time, the 5G loop bandwidth is set to $10 \mathrm{~Hz}$.

- MULTI $10 \mathrm{~Hz}$ : A complete channel is considered (LOS + multipath); the 5G loop bandwidth is set to $10 \mathrm{~Hz}$, this case permits to quantify the distortion due to the multipath 
- MULTI $1 \mathrm{~Hz}$ : A complete channel is considered (LOS + multipath); the 5G loop bandwidth is set to $1 \mathrm{~Hz}$, this case illustrates the importance of the $5 \mathrm{G}$ ranging module tuning.

Two cases are considered for GNSS; an AWGN case and a complete channel.

The positioning performances obtained using the previously describe navigation filters are studied and compared for several scenarios,

- $5 \mathrm{G}$ or GNSS standalone navigation solution and hybrid navigation solution. By doing so, the performances of a 5G navigation solution with respect to a GNSS navigation solution can be compared. The interest of the hybrid solution is to determine if the available GNSS measurements can improve the 5G standalone navigation solution.

- Propagation channel: AWGN, LOS and LOS plus multipath. The comparison between these 3 propagation channels permit to quantify the impact of the multipath on the navigation solution with respect to the LOS case only. The AWGN case permit to have a reference to be compared to.

- Fitting and over bounding distribution characterization. As presented in the third section, both methods have to be compared in order to select the more appropriate one and to provide requirements.

In section 3, two characterization have been derived, an over-bounding method and a fitting method. The characterization of the performances of the navigations filters must also be done for both methods in order to select the appropriate method.

These configurations are summarized in Table 4.

Table 4 - Cases summary

\begin{tabular}{|c|c|c|c|c|c|c|}
\hline \multirow{2}{*}{\multicolumn{2}{|c|}{ Case }} & \multicolumn{3}{|c|}{ 5G Propagation channel } & \multicolumn{2}{|c|}{ GNSS propagation channel } \\
\hline & & $\begin{array}{l}\text { Propagation channel } \\
\text { model }\end{array}$ & $\begin{array}{c}\text { Loop } \\
\text { bandwidth }\end{array}$ & $\begin{array}{c}\text { Characterization } \\
\text { used }\end{array}$ & Propagation channel model & $\begin{array}{c}\text { Characterization } \\
\text { used }\end{array}$ \\
\hline 1 & AWGN & $\begin{array}{l}\alpha^{k}(t, \tau) \\
=\delta\left(\tau-\tau_{0}\right)\end{array}$ & $B_{l}=10 \mathrm{~Hz}$ & $\begin{array}{c}99.9 \%-N(0, \sigma) \\
\text { Overbounding }\end{array}$ & $\alpha(t, \tau)=\delta\left(\tau-\tau_{0}\right)$ & $\begin{array}{c}99.9 \%-N(0, \sigma) \\
\text { Overbounding }\end{array}$ \\
\hline 2 & LOS & $\begin{array}{l}\alpha^{k}(t, \tau) \\
=\alpha^{k}(t) \\
\cdot \delta\left(\tau-\tau_{0}(t)\right)\end{array}$ & $B_{l}=10 \mathrm{~Hz}$ & $\begin{array}{c}99.9 \%-N(0, \sigma) \\
\text { Overbounding }\end{array}$ & \multirow{5}{*}{$\begin{array}{l}\alpha(t, \tau) \\
=\sum_{l=0}^{N} \gamma_{l}(t) e^{-j \Phi_{l}(t)} \delta(\tau \\
\left.-\tau_{l}(t)\right)\end{array}$} & \multirow{5}{*}{$\begin{array}{c}99.9 \%-N(0, \sigma) \\
\text { Overbounding }\end{array}$} \\
\hline 3 & $\begin{array}{l}\text { Multi- } \\
10 \mathrm{~Hz}\end{array}$ & $\begin{array}{l}\alpha^{k}(t, \tau) \\
=\sum_{l=0}^{L-1} \alpha_{l}^{k}(t) \\
\cdot \delta\left(\tau-\tau_{l}(t)\right) \\
0 \leq t \leq T_{\text {OFDM }}\end{array}$ & $B_{l}=10 H z$ & $\begin{array}{c}99.9 \%-N(0, \sigma) \\
\text { Overbounding }\end{array}$ & & \\
\hline 4 & $\begin{array}{l}\text { Multi- } \\
1 \mathrm{~Hz}\end{array}$ & $\begin{array}{l}\alpha^{k}(t, \tau)= \\
=\sum_{l=0}^{L-1} \alpha_{l}^{k}(t) \\
\cdot \delta\left(\tau-\tau_{l}(t)\right) \\
0 \leq t \leq T_{\text {OFDM }}\end{array}$ & $B_{l}=1 H z$ & $\begin{array}{c}99.9 \%-N(0, \sigma) \\
\text { Overbounding }\end{array}$ & & \\
\hline 5 & $\begin{array}{c}\text { Multi- } \\
10 \mathrm{~Hz}\end{array}$ & $\begin{array}{l}\alpha^{k}(t, \tau) \\
=\sum_{l=0}^{L-1} \alpha_{l}^{k}(t) \\
\cdot \delta\left(\tau-\tau_{l}(t)\right) \\
0 \leq t \leq T_{O F D M}\end{array}$ & $B_{l}=10 \mathrm{~Hz}$ & $\begin{array}{c}99.9 \%-N(0, \sigma) \\
\text { Fitting }\end{array}$ & & \\
\hline 6 & $\begin{array}{l}\text { Multi- } \\
1 \mathrm{~Hz}\end{array}$ & $\begin{array}{l}\alpha^{k}(t, \tau)= \\
=\sum_{l=0}^{L-1} \alpha_{l}^{k}(t) \\
\cdot \delta\left(\tau-\tau_{l}(t)\right) \\
0 \leq t \leq T_{\text {OFDM }}\end{array}$ & $B_{l}=1 \mathrm{~Hz}$ & $\begin{array}{c}99.9 \%-N(0, \sigma) \\
\text { Fitting }\end{array}$ & & \\
\hline
\end{tabular}


For each cases, the navigation filters considered are detailed in Table 5

Table 5 - Navigation filter configurations

\begin{tabular}{|c|c|}
\hline Filters & Measurements \\
\hline \multirow{3}{*}{ EKF } & 5G Standalone \\
\cline { 2 - 2 } & GNSS Standalone \\
\cline { 2 - 2 } & $5 \mathrm{G}$ GNSS Hybrid \\
\hline \multirow{2}{*}{ UKF } & $5 \mathrm{G}$ Standalone \\
\cline { 2 - 2 } & $5 \mathrm{G}$ GNSS Hybrid \\
\hline
\end{tabular}

\section{2 - Results}

In order to characterize the performances of the navigation filters, the figure of merit selected is the Root Mean Square Error (RMSE) of the position error along the $\mathrm{X}, \mathrm{Y}$ and $\mathrm{Z}$ axis expressed in the East North Up coordinate frame. The horizontal RMSE is also provided $\left(\mathrm{X}^{2}+\mathrm{Y}^{2}\right)$.

In Table 6 to Table 11, the RMSEs for the cases 1 to 6 are provided for the 5 navigation filters. The results are illustrated in Figure 27 to Figure 31. Figure 27 to Figure 30 illustrates the multipath cases, i.e. case 3 to 6;Figure 31 proposes a comparison of the over bounding and fitting characterization for the hybrid filters. These cases are judged more realistic and pertinent for the study.

Table 6 - case 1 - AWGN - 99. 9\% - N(0, $\sigma)$ overbounding

\begin{tabular}{|c|c|c|c|c|}
\hline & $\mathrm{X}$ & $\mathrm{Y}$ & $\mathrm{Z}$ & $\mathrm{X}^{2}+\mathrm{Y}^{2}$ \\
\hline EKF GNSS & 1.048 & 0.795 & 1.410 & 1.326 \\
\hline EKF 5G & 0.085 & 0.270 & 0.039 & 0.283 \\
\hline UKF 5G & 0.052 & 0.015 & 0.090 & 0.054 \\
\hline EKF HYBRID & 0.230 & 0.054 & 0.475 & 0.236 \\
\hline UKF HYBRID & 0.282 & 0.287 & 0.415 & 0.403 \\
\hline
\end{tabular}

\begin{tabular}{|c|c|c|c|c|}
\hline & $\mathrm{X}$ & $\mathrm{Y}$ & $\mathrm{Z}$ & $\mathrm{X}^{2}+\mathrm{Y}^{2}$ \\
\hline EKF GNSS & 1.096 & 3.609 & 6.546 & 3.772 \\
\hline EKF 5G & 1.004 & 1.359 & 15.147 & 1.690 \\
\hline UKF 5G & 1.013 & 1.426 & 15.247 & 1.749 \\
\hline EKF HYBRID & 0.318 & 1.222 & 2.701 & 1.263 \\
\hline UKF HYBRID & 0.624 & 0.637 & 0.402 & 1.568 \\
\hline \multicolumn{7}{|c}{ Table 10 - case 5 - Multi - 99.9\% $-\boldsymbol{N}(\mathbf{0}, \boldsymbol{\sigma})$ fitting }
\end{tabular}

\begin{tabular}{|c|c|c|c|c|}
\hline & $\mathrm{X}$ & $\mathrm{Y}$ & $\mathrm{Z}$ & $\mathrm{X}^{2}+\mathrm{Y}^{2}$ \\
\hline EKF GNSS & 1.096 & 3.609 & 6.546 & 3.772 \\
\hline EKF 5G & 1.467 & 1.705 & 12.498 & 2.250 \\
\hline UKF 5G & 1.530 & 1.844 & 12.519 & 2.396 \\
\hline EKF HYBRID & 1.312 & 1.806 & 8.559 & 2.232 \\
\hline UKF HYBRID & 0.344 & 0.553 & 0.715 & 0.651 \\
\hline
\end{tabular}

Table 7 - case 2 - LOS - 99. $9 \%-N(0, \sigma)$ overbounding

\begin{tabular}{|c|c|c|c|c|}
\hline & $\mathrm{X}$ & $\mathrm{Y}$ & $\mathrm{Z}$ & $\mathrm{X}^{2}+\mathrm{Y}^{2}$ \\
\hline EKF GNSS & 1.096 & 3.609 & 6.546 & 3.772 \\
\hline EKF 5G & 0.149 & 0.264 & 0.162 & 0.303 \\
\hline UKF 5G & 0.124 & 0.043 & 0.254 & 0.1319 \\
\hline EKF HYBRID & 0.331 & 0.132 & 0.552 & 0.357 \\
\hline UKF HYBRID & 0.648 & 0.336 & 0.814 & 0.696 \\
\hline
\end{tabular}

\begin{tabular}{|c|c|c|c|c|}
\hline & $\mathrm{X}$ & $\mathrm{Y}$ & $\mathrm{Z}$ & $\mathrm{X}^{2}+\mathrm{Y}^{2}$ \\
\hline EKF GNSS & 1.096 & 3.609 & 6.546 & 3.772 \\
\hline EKF 5G & 0.683 & 0.579 & 4.889 & 0.895 \\
\hline UKF 5G & 0.648 & 0.478 & 4.955 & 0.805 \\
\hline EKF HYBRID & 0.444 & 0.701 & 2.475 & 0.830 \\
\hline UKF HYBRID & 0.718 & 0.458 & 0.909 & 0.852 \\
\hline
\end{tabular}

\begin{tabular}{|c|c|c|c|c|}
\hline & $\mathrm{X}$ & $\mathrm{Y}$ & $\mathrm{Z}$ & $\mathrm{X}^{2}+\mathrm{Y}^{2}$ \\
\hline EKF GNSS & 1.096 & 3.609 & 6.546 & 3.772 \\
\hline EKF 5G & 0.723 & 0.714 & 16.351 & 1.016 \\
\hline UKF 5G & 0.720 & 0.660 & 16.303 & 0.977 \\
\hline EKF HYBRID & 0.679 & 0.658 & 3.768 & 0.946 \\
\hline UKF HYBRID & 0.644 & 0.546 & 0.980 & 0.844 \\
\hline
\end{tabular}



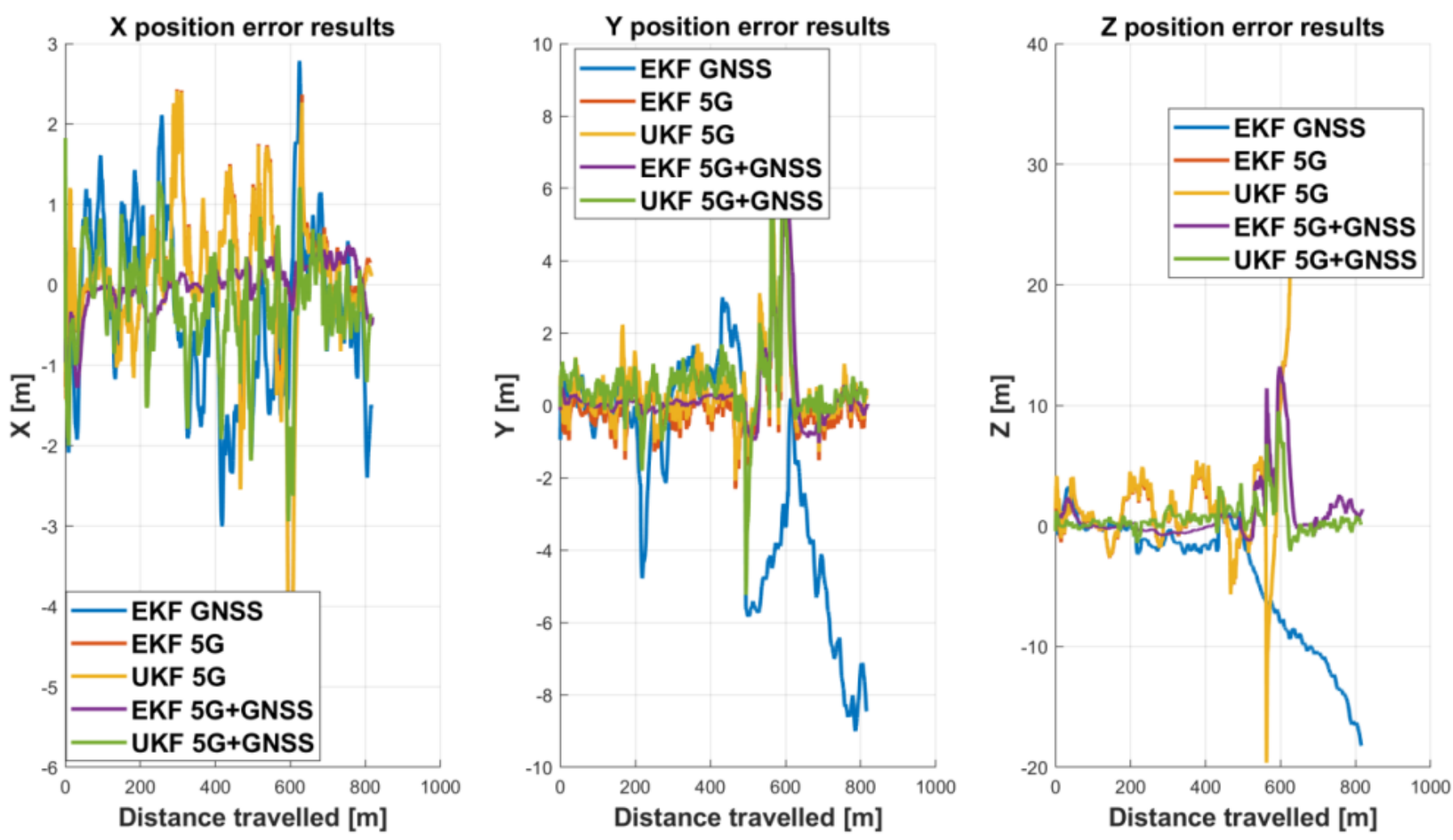

Figure 27 - Errors on position - Case 3
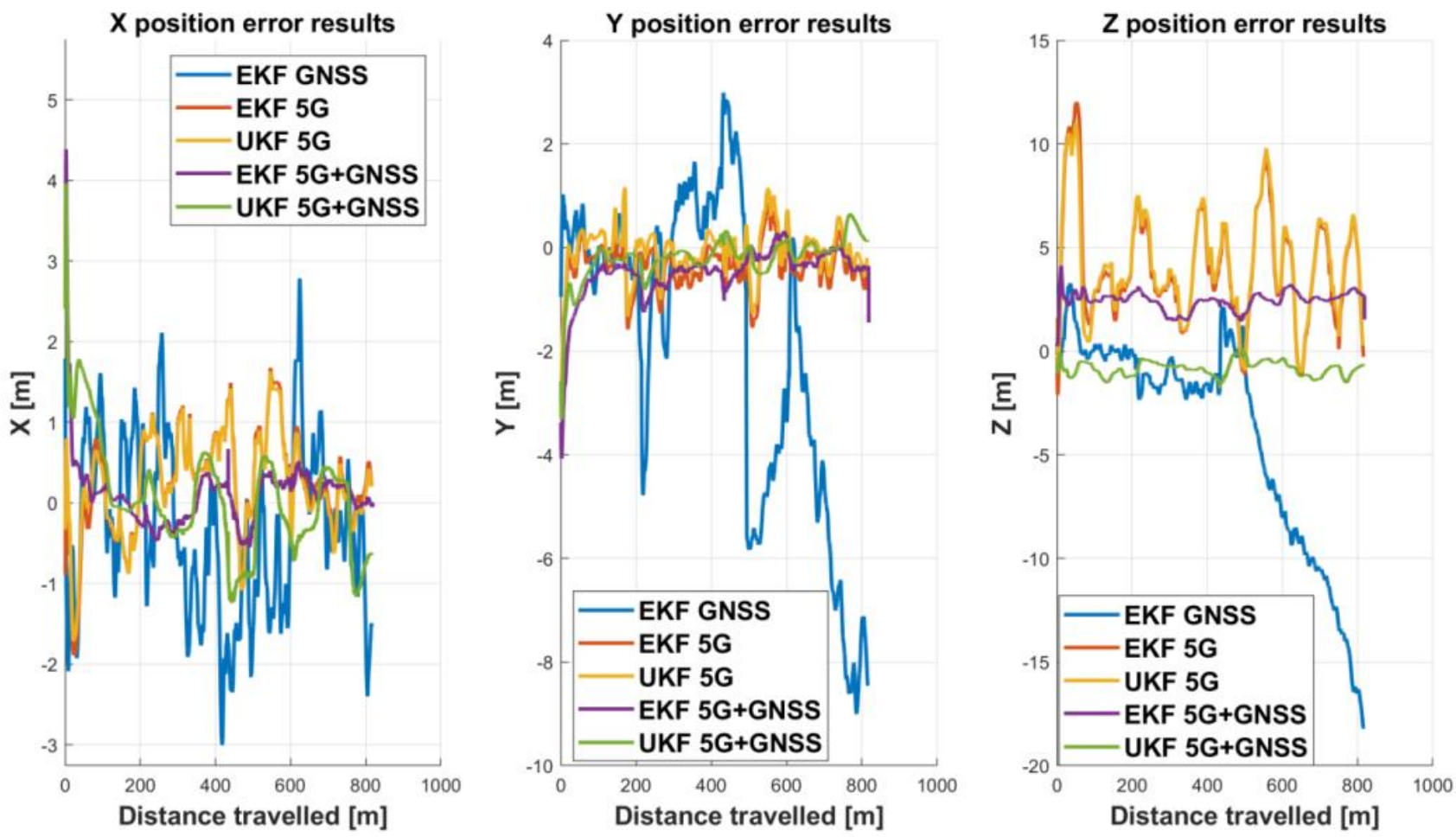

Figure 28 - Errors on position - Case 4 

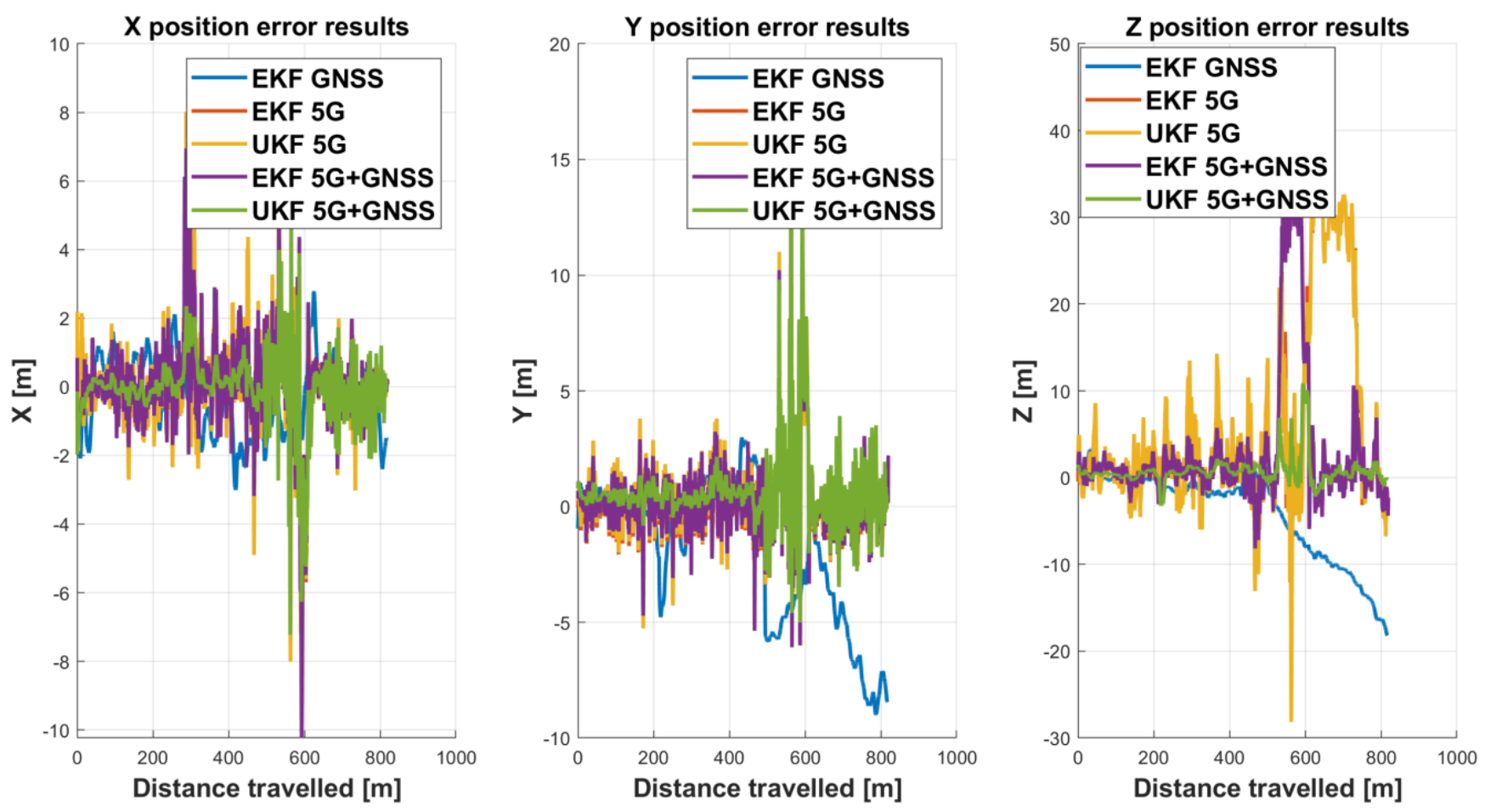

Figure 29 - Errors on position - Case 5
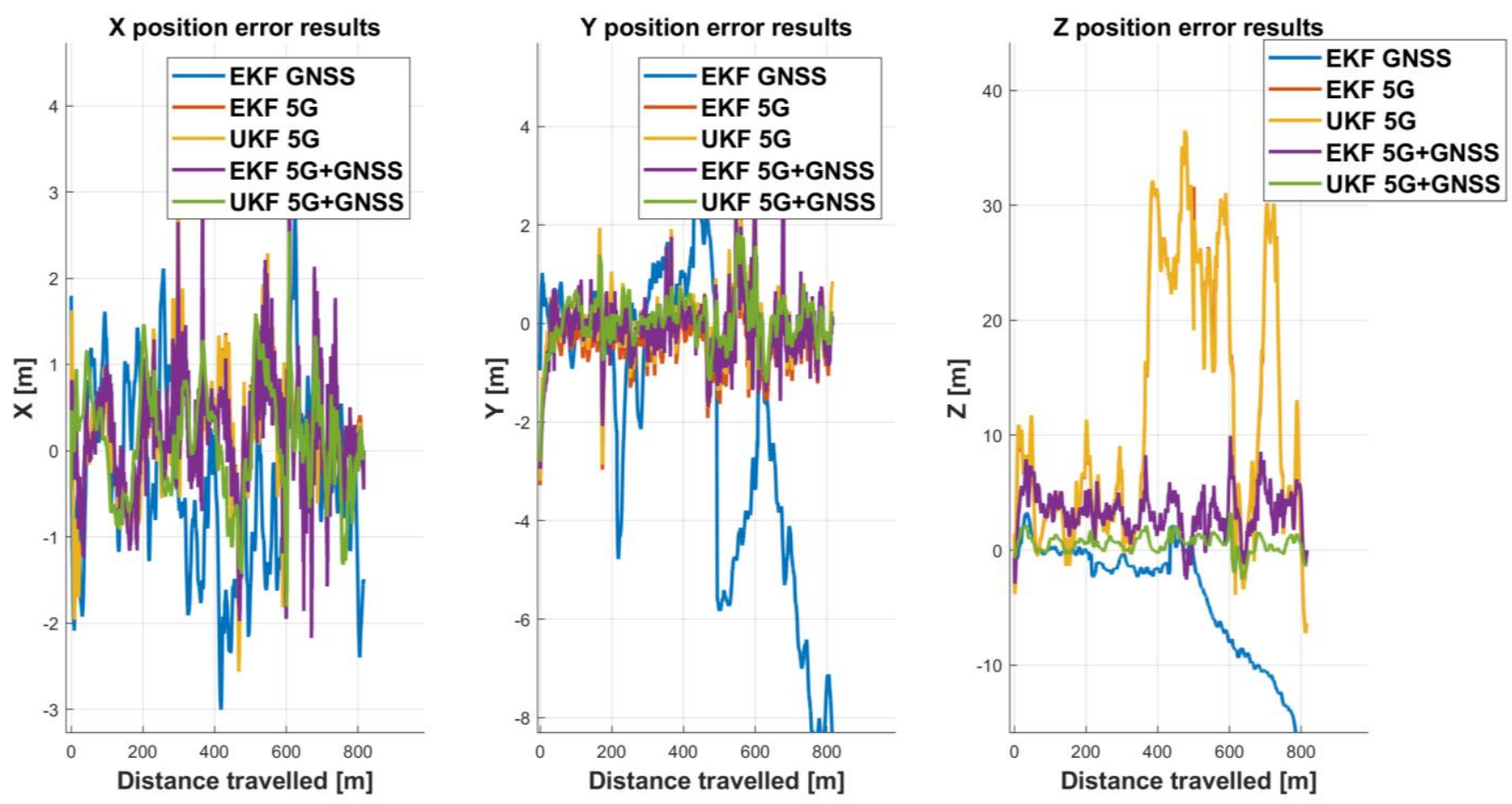

Figure 30 - Errors on position - Case 6 

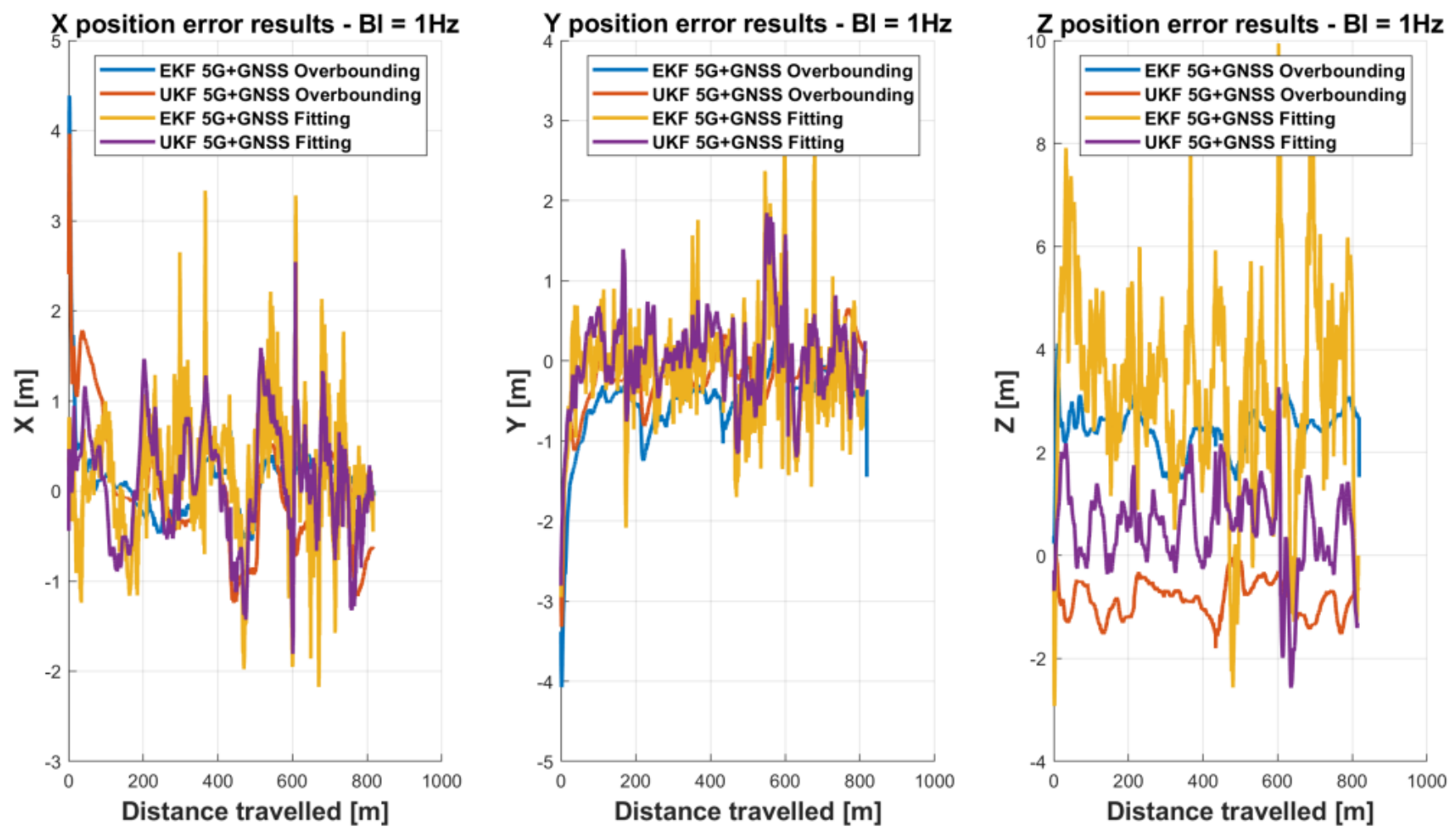

Figure 31 - Comparison of the characterization methods

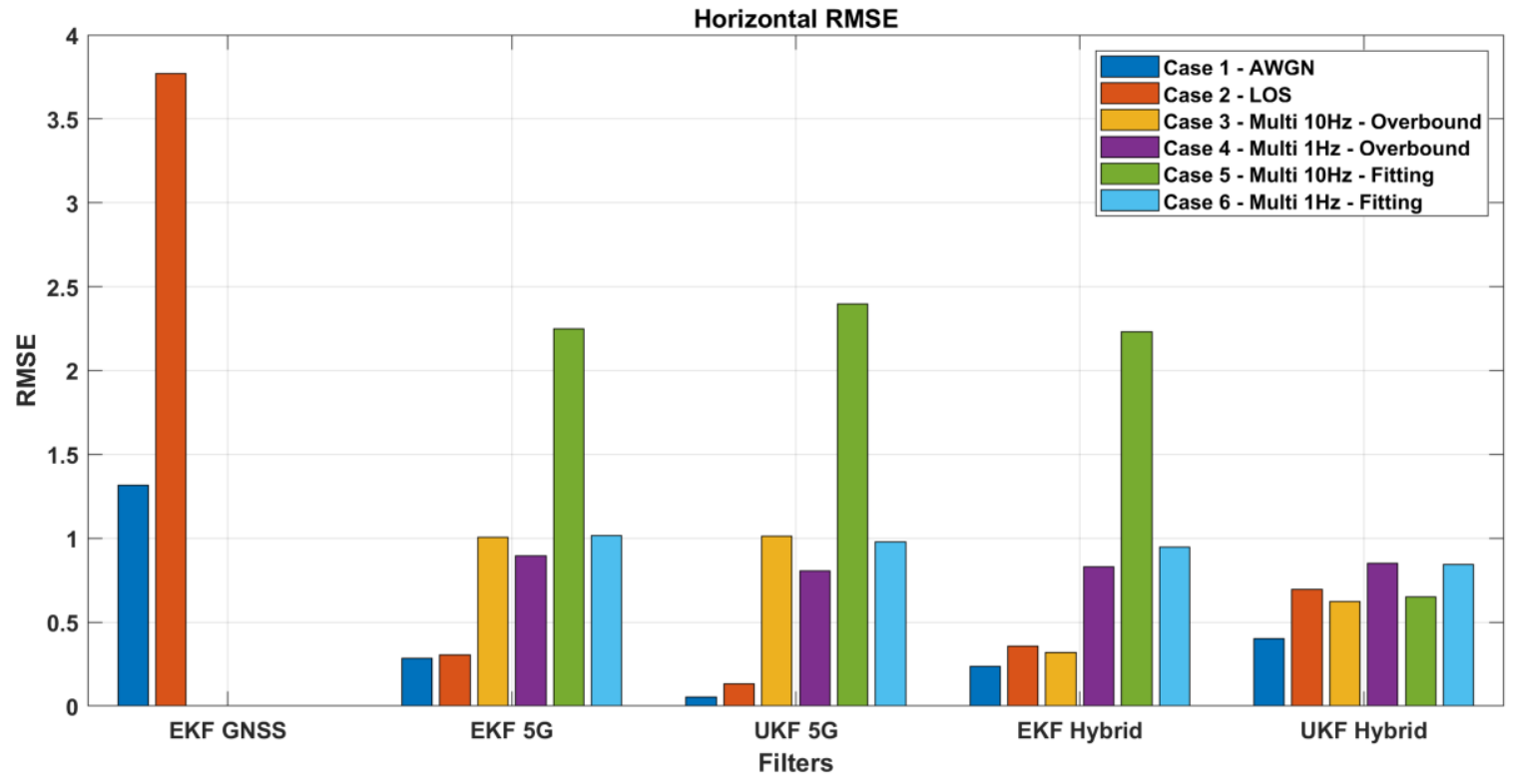

Figure 32 - Histogram of the RMSE on the horizontal plane 


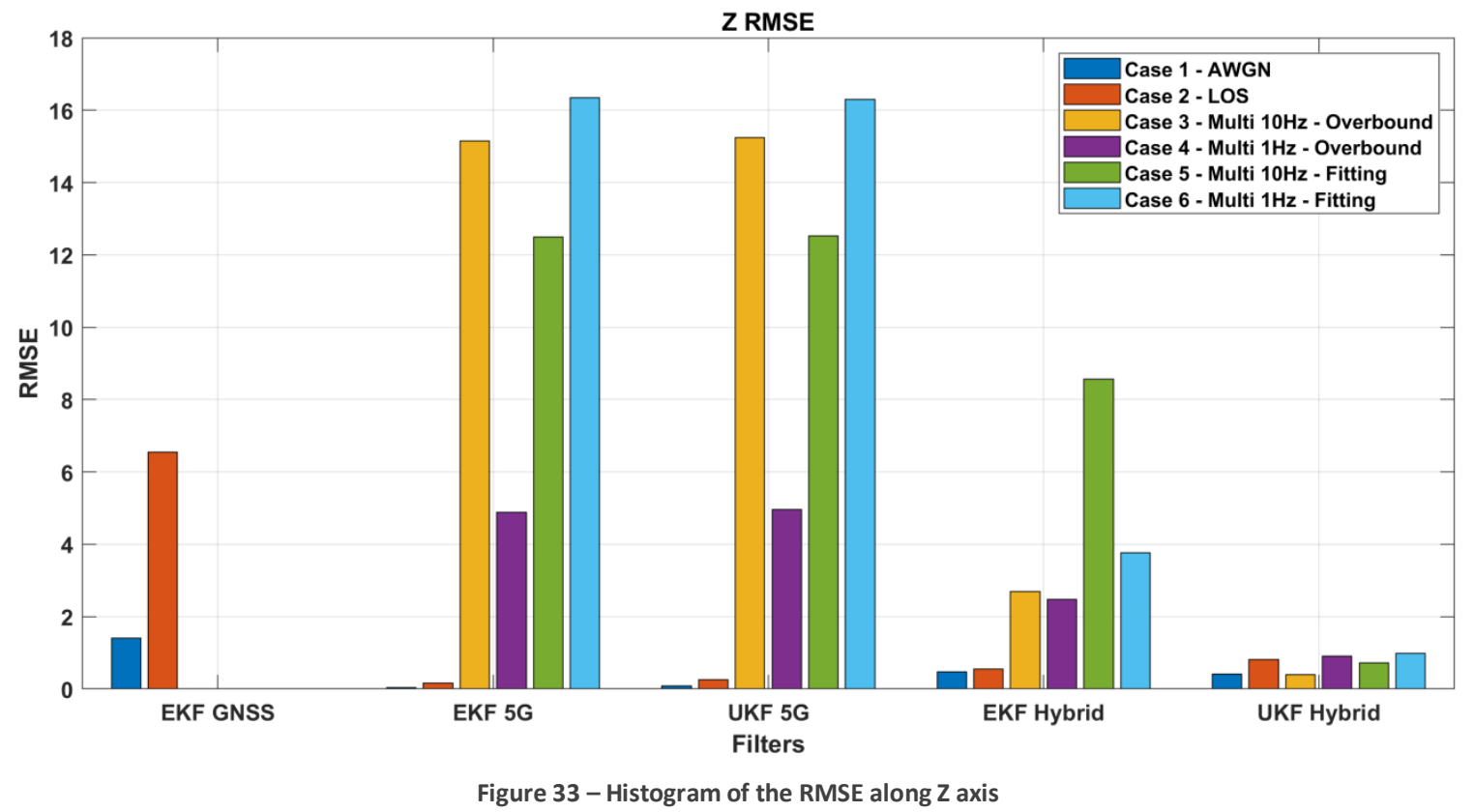

From all these tables and figures several observations can be made.

First, regarding the performances of $5 \mathrm{G}$ standalone solution, there is no significant differences between the UKF and the EKF. This can be observed basically in the each of the six tables (Table 6 to Table 11) by comparing the EKF 5G and the UKF 5G lines. Actually, this was predictable since for linear models UKF and EKF give the same results. Another important observation is the performances along the $\mathrm{Z}$ axis. The RMSE reaches $15 \mathrm{~m}$ and $5 \mathrm{~m}$ for the multipath cases for $10 \mathrm{~Hz}$ and $1 \mathrm{~Hz}$ loop bandwidths respectively; see Table 8 and Table 9. This behavior was also predictable since all BSs are at the same height; thus the geometry along the $\mathrm{Z}$ axis is not good enough. This can be compared to the Dilution Of Precision (DOP) used for GNSS.

Second, it must be noted that the design of the $5 \mathrm{G}$ ranging module is also important. The performances of the navigation filter using a $1 \mathrm{~Hz}-\mathrm{DLL}$ are better than with a $10 \mathrm{~Hz}-\mathrm{DLL}$. It can be seen from Figure 32, the cases 3 and 5 bars are larger than the case 4 and 6 bars particularly for the 5G standalone and the EKF hybrid navigation solutions. These observations are important since a $5 \mathrm{G}$ ranging module is still to be designed.

Third, the hybrid navigation solution provides better solution than the standalone navigation solutions. In Figure 34 the number of measurements along the trajectory is provided. The number is obtained for a QuaDRiGa complete channel and a SCHUN channel. As illustrated in Figure 34, using only GNSS would not permit to compute a positioning solution since less than 4 satellites are in view after $500 \mathrm{~m}$. This can also be seen from Figure 27 and Figure 28 with the increase of the $\mathrm{Y}$ and $\mathrm{Z}$ errors after $500 \mathrm{~m}$. Thus the use of the $5 \mathrm{G}$ signals improves the positioning solution availability in urban canyon. Nevertheless, by comparing the performances of the $5 \mathrm{G}$ standalone navigation solution and the hybrid navigation solution it can be seen than even if there are few GNSS satellites in view they still permit to improve the positioning precision. 


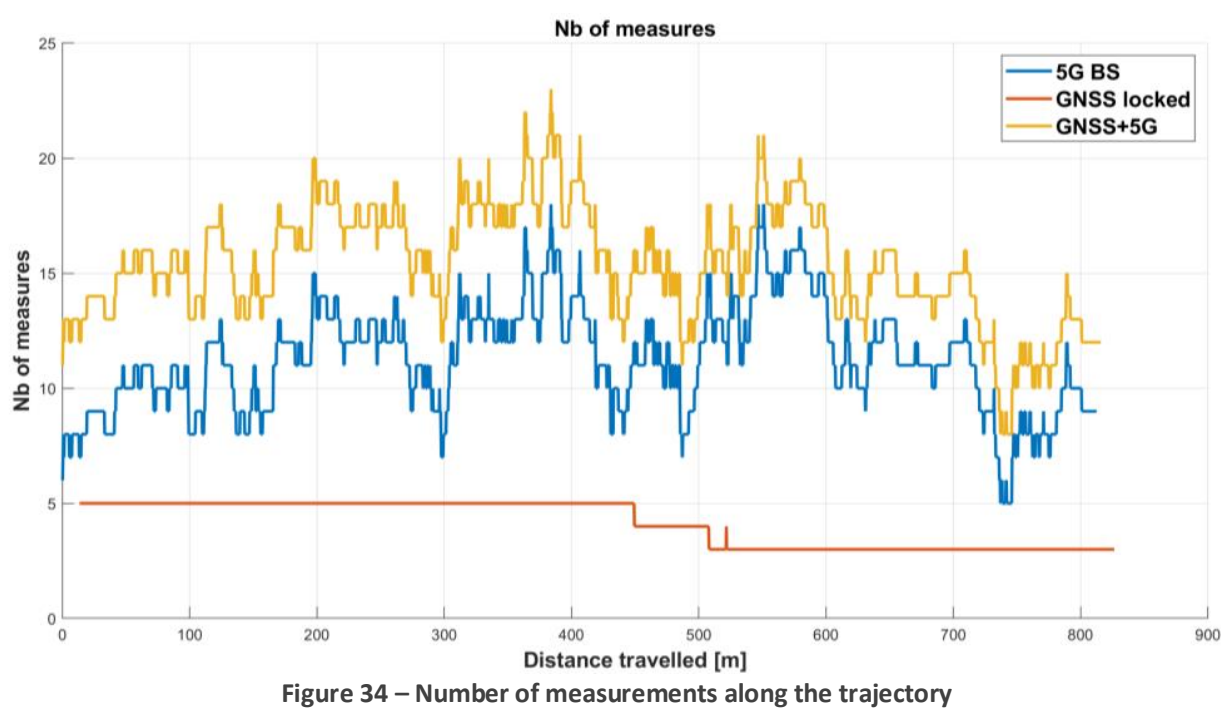

Fourth, the performances obtained depending on the pseudo range statistical characterization method, over-bounding and fitting methods, are studied. By comparing Table 8 and Table 10, the performances obtained with the fitting method seem to be worse than with the over bounding method; at least along the $\mathrm{X}$ and $\mathrm{Y}$ axis and for the standalone 5G navigation filters (UKF and EKF) and the hybrid EKF. By comparing Table 9 and Table 11, even if the difference is thinner than with Table 8 and Table 10, the over bounding slightly outperforms the fitting method.

Finally, by comparing the last 2 lines of Table 8 , Table 9, Table 10 and Table 11, it can be seen that the performances of the UKF hybrid navigation solution is slightly better than the performances of the EKF hybrid navigation solution. Nevertheless, it is important to remind that, due to numerical issues, the availability of the EKF is higher than the UKF.

According to these observations several recommendations can be provided. First of all, the use of $5 \mathrm{G}$ measurements is highly recommended in constrained environment when there is a lack of GNSS measurements. If some GNSS measurements are available, a hybrid navigation solution is recommended since better performances, especially in the $\mathrm{Z}$ axis, can be expected. The tuning of the $5 \mathrm{G}$ ranging module is important, according to the study a $1 \mathrm{~Hz}$ DLL loop bandwidth is recommended. The pseudo range characterization has also a great importance and for the application the over bounding method provides better performances. Finally, the UKF is recommended with respect to the EKF.

\section{CONCLUSION AND FUTURE WORK}

The objectives of the article was to realistically characterize GNSS and 5G pseudo range measurement mathematical models and to develop hybrid navigation modules exploiting/adapted to the derived pseudo range measurements mathematical models. The pseudo range measurements mathematical models have been derived from a realistic simulator which integrates a typical GNSS receiver processing module and a typical 5G signal processing module proposition; moreover, in order to achieve a realistic characterization, the simulator implemented highly realistic propagation channels for GNSS, SCHUN [8], and for 5G, QuaDRiGa [9]. The hybrid navigation modules implemented and compared in this work are an Extended Kalman Filter (EKF) and an Unscented Kalman Filter (UKF).

This article has derived the correlator output mathematical models of $5 \mathrm{G}$ received signals for realistic time-evolving propagation channel models. The mathematical models of GNSS correlator outputs for realistic time-evolving propagation channel models have also been derived.

In order to derive accurately these mathematical models; the propagation channel selection for both GNSS and 5G systems has been made: SCHUN has been selected for GNSS systems and QuaDRiGa for 5G systems. The pseudo ranges measurements have been statistically characterized in those environments.

Finally hybrid navigation modules using both GNSS and 5G signals have been derived. The focus has been made on two particular filters: the EKF and the UKF. Both filters assume Gaussian errors; a particular attention has been given to the design of these Gaussian errors. The driven idea is that if the Gaussian distribution is overestimated or underestimated, the filters performances will be suboptimal.

Consequently, two methods to characterize the errors have been designed namely a fitting and an over-bounding method. In order to choose the right on, both have been used in the standalone and hybrid navigation filters. In particular the application envisioned in the paper, the over bounding method seems more promising than the fitting one. 
Standalone 5G and GNSS navigation filters have been compared to hybrid navigation filters. The scenario envisioned in the paper can be seen as an urban canyon; thus, basically, no GNSS solution is available since less than 4 satellites are in view. Considering this, the use of $5 \mathrm{G}$ signals is interesting since enough Base Stations are in view and a positioning solution can be computed. Nevertheless, due to the bad geometry of the $5 \mathrm{G}$ systems, the position in altitude is bad. However, the use of both GNSS and 5G signals, in hybrid navigation filters permits to overcome this issue and to obtain a positioning solution accurate $(<2 \mathrm{~m}$ errors) and continue.

The development of other filters, such as Particle Filters (PF), could now be implemented. The main advantage of PF is that they do not rely on a Gaussian PDF approximation contrary to Kalman Filters; an a-priori information on the distributions must be provided, and such information has been derived in this study. Indeed, the study on the characterization of the pseudo ranges errors has shown that the PDF obtained with QuaDRiGa are not Gaussian. Thus, by taking into account the true distribution for the PF better navigation filters performances could be obtained. Nevertheless, the complexity of such implementation will be higher.

\section{ACKNOWLEDGMENTS}

This work is supported by Thales Alenia Space and by the French Spatial Agency.

\section{REFERENCES}

[1] D. Serant, D. Kubrak, M. Monnerat, G. Artaud and L. Ries, "Field test performance assessment of GNSS/INS ultra-tight coupling scheme targeted to mass-market applications," in 2012 6th ESA Workshop on Satellite Navigation Technologies (Navitec 2012) \& European Workshop on GNSS Signals and Signal Processing, 2012.

[2] R. Broquet, N. Perrimon and B. Polle, "HiNAV Inertial/GNSS Hybrid Navigation Dystem for launchers and re-entry vehicles," in 2010 5th ESA Workshop on Satellite Navigation Technologies and European Workshop on GNSS Signals and Signal Processing (NAVITEC), 2010.

[3] K. Shamaei, J. Khalife and Z. Kassas, "Exploiting LTE Signals for Navigation: Theory to Implementation," 2018.

[4] C. Mensing, S. Plass and A. Damman, "Synchronization algorithms for Positionning with OFDM Communication Signals," 2007.

[5] C. Mensing and A. Dammann, " Positioning with OFDM based communications systems and GNSS in critical scenarios," 2008.

[6] E. Staudinger, C. Klein and S. Sand, "A generic OFDM based TDoA positioing testbed with interference mitigation for subsample delay estimation," 2011.

[7] P. Thevenon, D. Serant, O. Julien and C. Macabiau, "Positionning using mobile TV based on DVB-SH standard," 2014.

[8] D. Serant, P. Thevenon, O. Julien and C. Macabiau, "Development and validation of an OFDM/DVB-T sensor for positioning," 2010.

[9] J. Talvitie, M. Koivisto, T. Levanan, M. Valkama, G. Destino and H. Wymeersch, "High-Accuracy Joint Position and Orientation Estimation in Sparse 5G mmWave channel," Proc. IEEE International Conference on Communications (ICC), 2019.

[10] A.-M. Tobie, A. Garcia-Pena, P. Thevenon and M. Aubault, "Processed 5G Signals Mathematical Models for Positioning considering a Non-Constant Propagation Channel.," in VTC 2019-Fall2019 IEEE 90th Vehicular Technology Conference, Honolulu, Sep 2019.

[11] 3GPP, "3GPP - The mobile Broadband Standard," [Online]. Available: https://www.3gpp.org/DynaReport/38-series.htm.

[12] A. Tobie, A. Garcia-Pena, P. Thevenon, D. Serant, M. Aubault and F. Marmet, "Mathematical Models and Statistics of Processed 5G Signals for Ranging Based Positioning for a Realistic Propagation Channel," Navigation: journal of the institute of navigation, Submitted in August 2020 - Review.

[13] R. W. H. Jr. and N. González-Prelcic, "Fundamentals of mmWave Communication," in Joint IEE and Eurasip summer school on signal processing for $5 G$ wireless access, , 2016.

[14] E. Björnson, "Advanced Signal Processing for Massive MIMO," in Joint IEEE SPS and EURASIP Summer School on Signal Processing for $5 G$ Wireless Access, 2017. 
[15] S. Mumtaz, J. Rodriguez and L. Dai, mmwave massive MIMO: a paradigm for 5G, 2016.

[16] Fraunhofer Heinrich Hertz Institute, "Quasi Deterministic Radio Channel Generator User Manual and Documentation," August 2017.

[17] M. Ait Ighil, "Enhanced physical-statistical simulator of the land mobile satellite channel for multipath modelling applied to satellite navigation systems," 2013.

[18] D. Serant, P. Thevenon, O. Julien and C. Macabiau, "Development and validation of an OFDM/DVB-T sensor for positioning," 2010.

[19] P. Thevenon, D. Serant and al, "Positionning using mobile TV based on DVB-SH standard," 2014.

[20] 3GPP, "TS 38.211 Physical channels and modulation".

[21] 3GPP, "TS. 38.213 Physical layer procedures for control," 2020.

[22] 3GPP, "TR 38.901 - Study on channel model for frequencies from 0.5 to $100 \mathrm{GHz}, 2019$.

[23] J. J. S. Jr., P. Axelrad, B. W. Parkinson and P. Enge, Global Positioning System Volu me 1, 1996.

[24] H. A, Bayesian estimation and tracking: a practival guide, 2012.

[25] M. Rhudy, Y. Gu, J. Gross and M. R. Napolitano1, "Evaluation of Matrix Square Root Operations for UKF within a UAV GPS/INS Sensor Fusion Application," International Journal of Navigation and Observation, vol. $2011,2011$. 